\title{
Evolutionarily conserved regulation of embryonic fast-twitch skeletal muscle differentiation by Pbx factors
}

Gist H. Farr, III ${ }^{1}$, Bingsi Li ${ }^{2,3}$, Maurizio Risolino ${ }^{2,4}$, Nathan M. Johnson ${ }^{1,5}$, Zizhen Yao ${ }^{6,7}$, Robert M. Kao ${ }^{1,8}$, Mark W. Majesky ${ }^{1,9,10}$, Stephen J. Tapscott ${ }^{6}$, Licia Selleri ${ }^{2,4}$, and Lisa Maves ${ }^{1,9^{*}}$

${ }^{1}$ Center for Developmental Biology and Regenerative Medicine, Seattle Children's Research Institute, Seattle, WA, USA

${ }^{2}$ Department of Cell and Developmental Biology, Weill Medical College of Cornell University, New York, NY, USA

${ }^{3}$ Current address: Burning Rock Biotech, Guangzhou, People's Republic of China

${ }^{4}$ Current address: Program in Craniofacial Biology; Institute of Human Genetics; Eli and Edythe Broad Center of Regeneration Medicine and Stem Cell Research; Department of Orofacial

Sciences and Department of Anatomy; University of California at San Francisco, San Francisco, CA, USA

${ }^{5}$ Current address: Division of Immunology, Tulane National Primate Research Center, Tulane University School of Medicine, Covington, LA, USA

${ }^{6}$ Division of Human Biology, Fred Hutchinson Cancer Research Center, Seattle, WA, USA

${ }^{7}$ Current address: Department of Informatics and Data Science, Allen Institute for Brain Science,

Seattle, WA, USA

${ }^{8}$ Current address: College of Arts \& Sciences, Heritage University, Toppenish, WA, USA

${ }^{9}$ Department of Pediatrics, University of Washington, Seattle, WA, USA

${ }^{10}$ Department of Pathology, University of Washington, Seattle, WA, USA 
*Corresponding author: Lisa Maves, lmaves@u.washington.edu, Center for Developmental

Biology and Regenerative Medicine, Seattle Children's Research Institute, 1900 Ninth Avenue, Seattle, WA 98101 USA. 


\section{Summary}

Vertebrate skeletal muscles are composed of both slow-twitch and fast-twitch fiber types. How the differentiation of distinct fiber types is activated during embryogenesis is not well characterized. Skeletal muscle differentiation is initiated by the activity of the myogenic basic helix-loop-helix (bHLH) transcription factors Myf5, Myod1, Myf6, and Myog. Myod1 functions as a muscle master regulatory factor and directly activates muscle differentiation genes, including those specific to both slow and fast muscle fibers. Our previous studies showed that Pbx TALE-class homeodomain proteins bind with Myod1 on the promoter of the zebrafish fast muscle gene mylpfa and are required for proper activation of mylpfa expression and the fasttwitch muscle-specific differentiation program in zebrafish embryos. Pbx proteins have also been shown to bind regulatory regions of muscle differentiation genes in mammalian muscle cells in culture. Here, we use new zebrafish mutant strains to confirm the essential roles of zebrafish $\mathrm{Pbx}$ factors in embryonic fast muscle differentiation. Furthermore, we examine the requirements for $P b x$ genes in mouse embryonic skeletal muscle differentiation, an area that has not been investigated in the mammalian embryo. Removing $\mathrm{Pbxl}$ function from skeletal muscle in $M y f 5^{\mathrm{Cre} /+;} \mathrm{Pbx} \mathrm{I}^{\mathrm{fl} f l}$ mouse embryos has minor effects on embryonic muscle development. However, concomitantly deleting $\mathrm{Pbx2}$ function in $\mathrm{Myf5}^{\mathrm{Cre} /+} ; \mathrm{Pbx}{ }^{f l f f l} ; \mathrm{Pbx} 2^{-/-}$mouse embryos causes delayed activation and reduced expression of fast muscle differentiation genes. In the mouse, $P b x 1 / P b x 2$-dependent fast muscle genes closely match those that have been previously shown to be dependent on murine Sixl and Six4. This work establishes evolutionarily conserved requirements for $\mathrm{Pbx}$ factors in embryonic fast muscle differentiation. Our studies are revealing how Pbx homeodomain proteins help direct specific cellular differentiation pathways. 
Keywords: $\mathrm{Pbx} 1, \mathrm{Pbx} 2, \mathrm{Pbx} 4$, Skeletal muscle, Fiber-type differentiation, Mouse, Zebrafish

\section{Introduction}

Skeletal muscle fiber diversity allows for a range of movements, activity and metabolism, but muscle fiber diversity can also affect susceptibility to muscle disease and muscle wasting (reviewed in Schiaffino and Reggiani, 2011; and Talbot and Maves, 2016). There are two main types of skeletal muscle fibers: slow twitch and fast twitch. Understanding how skeletal muscle fiber type differentiation is controlled is important for addressing skeletal muscle defects that occur in muscle aging, metabolic diseases, and muscular dystrophies (reviewed in Ciciliot et al., 2013; Ljubicic et al., 2014; and Talbot and Maves, 2016). Many processes have been described that regulate the plasticity of skeletal muscle fiber-type identities during post-natal periods (reviewed in Bassel-Duby and Olson, 2006; Blaauw et al., 2013; and Schiaffino and Reggiani, 2011). However, the mechanisms that establish muscle fiber-type identities during embryogenesis are less well understood.

Skeletal muscle differentiation is initiated in embryogenesis by the myogenic regulatory factor (MRF) family of basic helix-loop-helix (bHLH) transcription factors: Myf5, Myod1, Myf6, and Myog (reviewed in Hernández-Hernández et al, 2017; Lassar, 2017; and Zammit, 2017). The activity of these factors is highly conserved. In both mice and zebrafish, the MRFs are required for skeletal muscle myogenesis (reviewed in Buckingham and Vincent, 2009; and Rossi and Messina, 2014). In particular, Myod1 functions as a muscle master regulatory factor and controls skeletal muscle differentiation through DNA binding and transcriptional activation 
of broad gene expression programs, including genes encoding skeletal muscle contractile proteins (Blais et al., 2005; Cao et al., 2006; Cao et al., 2010; Pliner et al., 2018; Soleimani et al., 2012). In the differentiation of specific muscle fiber types, Myod1 has been associated with promoting fast muscle differentiation. In mouse muscle, Myod1 is expressed more strongly in fast fibers than in slow fibers, and gain- and loss-of-function experiments in mice and zebrafish have shown that Myod1 promotes fast fiber types (Ekmark et al., 2007; Hammond et al., 2007; Hinits et al., 2011; Hughes et al., 1997; Macharia et al., 2010; Maves et al., 2007; Seward et al., 2001). However, these and other studies have found that Myod1 is also needed for slow fiber differentiation and directly activates both slow and fast muscle differentiation genes (Blais et al., 2005; Cao et al., 2006; Cao et al., 2010; Hinits et al., 2011; Hughes et al., 1997; Maves et al., 2007; Seward et al., 2001; Soleimani et al., 2012). Myod1's DNA binding and transcriptional activity can be modulated, positively and negatively, by many other transcription factors (reviewed in Berkes and Tapscott, 2005; Fong and Tapscott, 2013; and Talbot and Maves, 2016). Understanding how the activity of MRFs such as Myod1 is regulated during embryogenesis should provide insight into skeletal muscle fiber type specification (Talbot and Maves, 2016). We already understand some components of a transcriptional network used to activate skeletal muscle fiber-type specification during embryogenesis (reviewed in Jackson and Ingham, 2013; and Rossi and Messina, 2014). Transcription factors of the Six homeodomain family are needed to promote MRF gene expression and muscle differentiation in both mice and zebrafish embryos (Bessarab et al., 2008; Grifone et al., 2005; Niro et al., 2010; Nord et al., 2013; O’Brien et al., 2014; Richard et al., 2011; Talbot et al., 2019). In mouse embryos with compound loss of Sixl;Six4, and in zebrafish embryos with morpholino knock-down of sixla and sixlb, fast muscle gene expression is reduced in the early myotome, whereas expression of slow muscle 
genes is largely not affected (Bessarab et al., 2008; Niro et al., 2010; O’Brien et al., 2014).

However, in zebrafish embryos with genetic deletion of all four zebrafish sixl and six4 genes, migratory muscle precursor cells do not undergo proper specification or migration, but embryonic fast muscle in the trunk still forms normally (Talbot et al., 2019). The Sox6 transcription factor promotes fast muscle differentiation by repressing slow muscle differentiation in zebrafish embryos and in mouse embryos (Hagiwara et al., 2005; Hagiwara et al., 2007; Jackson et al., 2015; von Hofsten et al., 2008). In zebrafish, the transcription factor Prdm1a acts as a critical switch in embryonic muscle fiber-type specification. Prdm1a directly represses fast muscle differentiation genes and also represses sox6 expression, thereby promoting slow muscle differentiation in zebrafish (von Hofsten et al., 2008). However, even though Prdm1 is expressed in the embryonic mouse myotome, the onset of myogenesis and fast and slow muscle differentiation appear to proceed normally in Prdm1 mutant mouse embryos (Vincent et al., 2012). Thus, while there are many conserved aspects of muscle fiber-type specification and differentiation, there also appear to be differences between zebrafish and mouse in the regulation of embryonic muscle fiber type.

We previously showed, in zebrafish embryos, that Pbx TALE-class homeodomain proteins are required for the fast muscle differentiation program (Maves et al., 2007; Yao et al., 2013). In zebrafish embryos lacking Pbx proteins through antisense morpholino knockdown, activation of fast muscle differentiation genes, such as mylpfa and atp $2 a l a$, is delayed and, even upon activation, these genes show reduced expression (Maves et al., 2007; Yao et al., 2013). Studies in zebrafish and mouse embryos, as well as in mammalian cell culture models, have shown that Pbx proteins bind with Myod1 on the promoters of a subset of Myod1 target genes, including Myog and mylpfa (Berkes et al., 2004; Cho et al., 2015; de la Serna et al., 2005; 
Dell'Orso et al., 2016; Heidt et al., 2007; Maves et al., 2007; Pliner et al., 2018). Pbx proteins can bind silent Myod1 target gene promoters prior to Myod1 binding and muscle differentiation, suggesting the potential for Pbx proteins to function as pioneer factors in skeletal muscle differentiation (Berkes et al., 2004; Cho et al., 2015; de la Serna et al., 2005; Dell'Orso et al., 2016; for discussion of Pbx proteins as pioneer factors, see Grebbin and Schulte, 2017 and Selleri et al., 2019). $P b x$ genes are broadly expressed during embryogenesis and are required for many aspects of mouse embryo development (reviewed in Moens and Selleri, 2006; and Selleri et al., 2019). However, these previous studies have not directly addressed the requirements for Pbx proteins in mammalian skeletal muscle development. Considering that the mechanisms underlying muscle fiber-type differentiation can vary between mice and zebrafish, we tested whether Pbx proteins are required for fast muscle differentiation during mouse embryogenesis.

Here we generate new zebrafish mutant strains to confirm that zebrafish embryos lacking Pbx expression show reduced and delayed activation of fast muscle differentiation genes. Furthermore, we investigate the requirements for Pbx factors in early mouse muscle development. We show that both $\mathrm{Pbx} 1$ and $\mathrm{Pbx} 2$ are broadly expressed in muscle precursors in embryonic mouse somites. We use a conditional knock-out approach to generate mouse embryos lacking both $\mathrm{Pbx} 1$ and $\mathrm{Pbx} 2$ in $M y f 5$-expressing muscle precursors. We uncover that $M y f 5^{\mathrm{Cre} /+} ; \mathrm{Pbx} \mathrm{I}^{f l f f l} ; \mathrm{Pbx} 2^{-/-}$embryos show reduced and delayed activation of fast muscle differentiation genes. Our findings demonstrate evolutionary conservation in the requirements for $\mathrm{Pbx}$ proteins in promoting activation of the fast muscle differentiation program during vertebrate embryogenesis.

\section{Materials and Methods}


Maintenance of animal strains and generation of zebrafish pbx2 mutant strain.

All experiments involving live zebrafish and mice were carried out in compliance with Seattle Children's Research Institute IACUC guidelines.

Zebrafish were raised and staged as previously described (Westerfield, 2000). Time (hpf) refers to hours post-fertilization at $28.5^{\circ} \mathrm{C}$. In some cases, embryos were raised for periods at $24^{\circ} \mathrm{C}$. For studies prior to $24 \mathrm{hpf}$, somite (s) number was used for staging, and mutant and control embryos were somite-stage matched. The wild-type stock and genetic background used was AB. The $p b x 4^{b 557}$ mutant strain was previously described and is likely a null allele (Pöpperl et al., 2000). $p b x 4^{b 557}$ genotyping was performed as previously described (Kao et al., 2015). The $p b x 2^{s c m 10}$ mutant strain was generated with CRISPR/Cas9, using procedures we have previously described (Supplemental Figure 1; Farr et al., 2018). The pbx2-targeting single-guide construct was made by annealing the following oligonucleotides and ligating the resulting fragment into BsaI-digested pDR274 (Hwang et al., 2013): 5' - [phos]TAGGTGTAGCCGGGCCAGAGAA 3' and 5' - [phos]AAACTTCTCTGGCCCGGCTACA - 3'. To genotype the $p b x 2^{s c m 10}$ strain, a dCAPS assay was designed (Neff et al., 1998) such that amplification of the mutant allele generated an XcmI site. The sequence of the $p b x 2$ CRISPR target site is shown in Supplemental Figure 1, along with the sequence of the $p b x 2^{s c m 10}$ allele and its conceptual translation. Genotyping primers are provided in Table 1.

The mouse strains used were previously described: the Pbxl knock-out allele (Selleri et al., 2001; hereafter called $\left.P b x 1^{-/-}\right)$, the $P b x l$ conditional allele ( $P b x 1^{\Delta n e o ; \Delta e x 3}$; Koss et al., 2012; hereafter called $P b x I^{f l}$ ), the $P b x 2$ knock-out allele (Selleri et al., 2004; hereafter called $P b x 2^{-/}$), 
and the Myf5 Cre allele (Myf5 $5^{\text {tm3(cre)Sor; }}$ Tallquist et al., 2000; hereafter called Myf5 ${ }^{\text {Cre }}$; kindly provided by P. Soriano). Intercrosses were used to combine strains, which were maintained on a largely C57BL/6 background. Mouse embryos were obtained through timed matings, designating E0.5 as the morning on the day a vaginal plug was observed. Genotyping was performed by PCR using genomic DNA isolated from embryonic sacs or tail tissue. Protocols for $P b x 1, P b x 1^{f l}$, $P b x 2$, and $M y f 5^{C r e}$ genotyping were previously described (Koss et al., 2012; Selleri et al., 2001; Tallquist et al., 2000). Genotyping primers are provided in Table 1.

Immunocytochemistry and RNA in situ hybridization

Zebrafish whole-mount RNA in situ hybridizations were performed on somite-stagematched embryos as previously described (Maves et al., 2007; Talbot et al., 2010). The following cDNA probes were used: krox-20 (egr2b-Zebrafish Information Network, Ruzicka et al., 2019; Oxtoby and Jowett, 1993); myog (Weinberg et al., 1996); smyhcl (Bryson-Richardson et al., 2005); atp2al (Maves et al., 2007); myll (Yao et al., 2013); mylpfa (formerly mylz2; Xu et al., 2000). Zebrafish embryos were imaged using a Leica TCS SP5 confocal microscope. Images were assembled using Adobe Photoshop.

For anti-Pbx1/Myf5 staining in mouse embryos, E11.5 embryos were fixed in $4 \%$ PFA/1X PBS overnight at $4^{\circ} \mathrm{C}$ and then dehydrated and stored in methanol at $-20^{\circ} \mathrm{C}$. Embryos were rehydrated using a methanol series performed at room temperature. Rehydrated embryos were washed in $1 \mathrm{X}$ PBST $(1 \mathrm{X}$ PBS $+0.1 \%$ Triton $\mathrm{X}-100)$, followed by a puncture of the dorsal end of the neural tube to facilitate antibody diffusion. Embryos were rinsed in 1X PBSDT (1X PBS, $1 \%$ DMSO, $0.1 \%$ Triton X-100) followed by permeabilization in $1 \mathrm{X}$ PBS $+2 \%$ TritonX100 for 30 minutes at room temperature. Permeabilized embryos were then rinsed in $1 \mathrm{X}$ PBSDT 
and blocked overnight in 10\% normal donkey serum in 1X PBSDT. 1:100 rabbit polyclonal antiMyf5 (IgG $\mathrm{IH}_{\mathrm{L}}$, Santa Cruz, sc-302) and 1:100 monoclonal mouse anti-Pbx1b (IgG 1 , Santa Cruz, 41.1, Jacobs et al., 1999) were incubated in 2\% normal donkey serum in 1X PBSDT for 2 days at $4^{\circ} \mathrm{C}$. Embryos were then washed for five hours at room temperature in $1 \mathrm{X}$ PBSDT. For secondary antibodies, Alexa 488 donkey anti-mouse $\mathrm{IgG}_{1}$ and Alexa 594 donkey anti-rabbit IgG $\mathrm{H}_{\mathrm{H}+\mathrm{L}}$ were used at 1:500 dilution in $2 \%$ normal donkey serum in $1 \mathrm{X}$ PBSDT overnight at $4^{\circ} \mathrm{C}$. After secondary antibody staining, embryos were washed in 1X PBSDT. After post-fixation, embryos were pre-incubated in $30 \%$ glycerol $+0.1 \%$ (v/v) $\beta$ Scale2 (4M urea, $50 \%$ glycerol in distilled water) for 45 minutes; then Clear $^{T 2}$ in $25 \%$ PEG/10\% formamide was used for 1 hour at room temperature, followed by overnight in 50\% PEG/20\% formamide. Cleared embryos were mounted in $0.5 \%$ agarose gel in water and settled until gel polymerized; then Clear $^{T 2}$ in $50 \%$ PEG/20\% formamide was applied and the specimen was overlain with a coverslip. Optical stacks were collected using an upright Olympus single photon confocal microscope (20x water dipping objective, NA=0.95). Image datasets were analyzed using Fiji/ImageJ software.

For anti-Pbx2/Myod1 staining in mouse embryos, E11.5 embryos were fixed in prechilled 4\% PFA/1X PBS for one hour. Fixed embryos were incubated in 30\% sucrose/1X PBS and embedded in OCT frozen blocks. 30-micron-thick sagittal sections were taken on a Leica cryostat machine. Cryosections were blocked with 3\% BSA/1\% normal donkey serum in a humidified slide box and subsequently incubated with anti-Pbx2 (1:100, polyclonal rabbit, Santa Cruz, G-20) and anti-Myod1 (5.8A, mouse monoclonal $\mathrm{IgG}_{1}$ ) diluted in block solution. After rinses in $1 \mathrm{X}$ PBS with $0.25 \%$ Triton $\mathrm{X}-100$, sections were then incubated with 1:500 Alexa 488 mouse $\operatorname{IgG}_{1}$ and Alexa 594 rabbit secondary antibodies in block solution for 1.5 hours at room temperature. Fluorescently labeled specimens were mounted in $2 \%$ n-propyl-gallate in $100 \%$ 
glycerol. Images were collected using a Leica SP5 inverted fluorescent confocal microscope with 20x air objective $(\mathrm{NA}=0.7)$ or 40x oil objective $(\mathrm{NA}=1.4)$. Fiji/ImageJ software was used for image analysis.

Mouse whole-mount RNA in situ hybridizations were performed on somite-stagematched embryos as previously described (Di Giacomo et al., 2006). Myog probe was synthesized from a full-length mouse Myog cDNA cloned into the BamHI site of pCS2+. Tnnc2 probe was synthesized from a $600 \mathrm{bp}$ cDNA fragment cloned into pCRII-TOPO. Atp2al probe was synthesized from a 1236bp cDNA fragment cloned into pCRII-TOPO. Myh6 probe was synthesized from a $1243 \mathrm{bp}$ cDNA fragment cloned into pCRII-TOPO. Additional cDNA probes for Myh3, Myll, Mylpf, Myl4, and Myh7 were generated by adding T7 promoters to PCRamplified cDNA products. Primers used to amplify cDNA probes are provided in Table 1.

\section{Quantitative (q) RT-PCR}

For mouse embryo qRT-PCR, total RNA samples were prepared from whole, individual, genotyped mouse embryos by disrupting the embryos in TRIzol (Ambion; Thermo Fisher Scientific) by trituration through a $16 \mathrm{G}$ needle followed by a $26 \mathrm{G}$ needle, after which the standard TRIzol protocol was followed. The isopropanol-precipitated RNA was solubilized in water and then further purified using an RNeasy Kit (Qiagen). 100 ng of RNA samples plus random hexamers were used in a reverse-transcriptase reaction with SuperScriptII Reverse Transcriptase (Invitrogen) or with the iScript cDNA Synthesis Kit (Bio-Rad). Real-time PCR was performed using SYBR Green (Bio-Rad) and an Applied Biosystems 7900HT System, or using the KAPA SYBR FAST kit (KAPA Biosystems) on a Bio-Rad CFX96 machine, according 
to the manufacturer's instructions. Expression levels of the genes of interest were normalized to Ornithine decarboxylase 1 (Odc1). For primers, see Table 1.

For each gene tested, a $\Delta \Delta \mathrm{C}_{\mathrm{t}}$ value was determined for each of the control/mutant pairs of sibling embryos. Two-tailed, unpaired Student's t-tests with Welch's correction were used to determine if the observed values differed significantly from the null hypothesis of $\Delta \Delta \mathrm{C}_{\mathrm{t}}$ values of 0 . The resulting $\mathrm{P}$ values are shown in the graphs in Figures 2 and 4 . To construct the graphs, the tables of $\Delta \Delta \mathrm{C}_{\mathrm{t}}$ values were log transformed to give fold differences $\left(2^{-\Delta \Delta \mathrm{Ct}}\right)$. The boxes for each gene in the graphs extend from the 25 th to 75 th percentiles, the whiskers are at the minimum and maximum, the bar within the box represents the median, and the "+" symbol represents the mean. Statistical analysis was performed and the plots were made in GraphPad Prism7.

RNA-seq library preparation and sequencing

Total RNA samples were obtained, using TRIzol Reagent (Life Technologies) and then cleaned using RNeasy Kit (Qiagen), from three $M y f 5^{C r e /+} ; P b x 1^{f l f l} ; P b x 2^{-/-}$mutants and three $P b x 1^{f l /+} ; P b x 2^{-/-}$control sibling whole mouse embryos at E11.5. Library preparation and sequencing was carried out by the FHCRC Genomics Shared Resource. Sequencing libraries were prepared from total RNA using the TruSeq RNA Sample Prep Kit (Illumina) according to the manufacturer's instructions. Library size distributions were validated using an Agilent 2100 Bioanalyzer. Additional library quality control, blending of pooled indexed libraries, and cluster optimization were performed using the QPCR NGS Library Quantization Kit (Agilent Technologies). RNA-seq libraries were pooled (4-plex) and clustered onto a flow cell lane using 
an Illumina cBot. Sequencing occurred on an Illumina HiSeq 2000 using a single-read, 100 base read length (SR100) sequencing strategy.

RNA-seq data processing and analysis

Image analysis and base calling were performed with Illumina’s Real Time Analysis v1.12 software. Files were demultiplexed of indexed reads and generated in FASTQ format using Illumina's CASAVA v1.8. software. Reads were removed that did not pass Illumina's base call quality threshold. Reads were aligned to mouse genome build mm9, using TopHat 1.4 (Trapnell et al., 2009). SAMtools v0.1.18 (Li et al., 2009) was used to sort and index the TopHat alignments. The gene expression profiles of embryos were compared using the Bioconductor package edgeR. Sequencing data will be available at NCBI GEO.

Functional annotation enrichment analysis was performed by submitting Ensemble Gene IDs from Table S1 and Table S2 to DAVID analysis (Huang da et al., 2009).

\section{Results}

Zebrafish mzpbx2;pbx4 mutant embryos show impaired activation of fast muscle differentiation

In zebrafish, the main $p b x$ genes expressed during early embryonic myogenesis stages are pbx2 and pbx4 (Farr et al., 2018; Waskiewicz et al., 2002). Our previous studies of Pbx functions in zebrafish skeletal muscle development used antisense morpholinos to knock down both $p b x 2$ and $p b x 4$ expression, and we showed that these $p b x 2-M O ; p b x 4-M O$ embryos have delayed 
activation and reduced expression of fast muscle genes (Maves et al., 2007; Yao et al., 2013). Here, we have used CRISPR to generate a loss of function allele of zebrafish $p b x 2$ (Materials and Methods; Supplemental Figure 1). We find that $p b x 2^{-/}$fish are homozygous viable as adults, similar to $P b x 2^{-/}$mice (Selleri et al., 2004). We crossed $p b x 2$ mutant fish with the established pbx4 mutant strain (Pöpperl et al., 2000) to generate a $p b x 2^{-/-} ; p b x 4^{+/-}$viable strain. Incrossing these fish generated $m z p b \times 2^{-/-} ; p b x 4^{-/-}$mutant embryos, thereby removing the maternal contribution of $p b x 2$ expression (Waskiewicz et al., 2002) in addition to the zygotic functions of $p b x 2$ and $p b x 4$. We find that $m z p b x 2^{-/} ; p b x 4^{-/-}$embryos almost completely lack krox 20 expression in the hindbrain (Fig. 1), as expected for zebrafish embryos lacking Pbx proteins (Waskiewicz et al., 2002). We examined fast and slow muscle development in $m z p b x 2^{-/} ; p b x 4^{-/}$embryos using RNA in situ hybridization (Fig. 1). As controls, we simultaneously collected embryos from incrosses of $p b x 4^{+/}$fish. We observe that the fast muscle genes atp $2 a 1$, myll, and mylpfa, as well as myog, show reduced expression in $m z p b x 2^{-/} ; p b x 4^{-/}$embryos compared to somite-stage matched controls (Fig. 1). Trunk skeletal muscle expression of the slow muscle gene smyhcl appears largely unaffected in $m z p b x 2^{-/} ; p b x 4^{-/}$embryos (Fig. 1). These results corroborate our previously described findings in pbx2-MO;pbx4-MO embryos (Maves et al., 2007; Yao et al., 2013). We observed no detectable effect on muscle development in $m z p b x 2^{-/-}$embryos and only subtle, variable effects on fast muscle gene expression in $p b x 4^{-/}$embryos (data not shown). These results confirm that $\mathrm{Pbx}$ proteins are needed for the proper activation of fast muscle differentiation genes in zebrafish embryogenesis.

\section{Loss of Pbxl has little effect on early mouse muscle differentiation}


In mouse, previous studies have shown that $P b x l$ is expressed broadly in embryonic somites and is required for multiple developmental programs (Capellini et al., 2006; Selleri et al., 2001; Selleri et al., 2019). To investigate the requirements for Pbx factors in mouse muscle differentiation, we examined mouse embryos lacking Pbx1 (Pbxl ${ }^{-/}$; Selleri et al., 2001). Using qRT-PCR, we find no significant effects on muscle fiber-type markers in E9.5 $\mathrm{Pbxl^{-/- }}$ embryos (Fig. 2A). In E11.5 $\mathrm{Pbxl}^{-/}$embryos, we find downregulation of Myog expression and upregulation of the slow muscle gene $M y h 7$, but no significant changes in the other muscle differentiation genes (Fig. 2B). Using RNA in situ hybridization, we observe some downregulation of Myog expression in $\mathrm{Pbxl}^{-/}$embryos at E11.5, consistent with our qRT-PCR results (Fig. 2C-D). These results suggest that loss of Pbx1 alone has subtle effects on early mouse muscle differentiation.

Mouse Pbx1 and Pbx2 are both expressed in embryonic skeletal muscle precursors

Previous studies have shown that both $P b x 1$ and $P b x 2$ mRNAs are expressed broadly in mouse somites at E8.5 (Capellini et al., 2006). We thus wanted to determine whether Pbx1 and $\mathrm{Pbx} 2$ proteins are expressed in mouse embryo somites, which contain the primary myotome, the precursors of early mouse muscle (Biressi et al., 2007). Using antibody staining, we observe broad somitic expression of Pbx1 and Pbx2 at E11.5, (Fig. 3A,C), and they both show nuclear localization (Fig. 3B,D). Pbx1 and Pbx2 show overlap with Myf5 and Myod1 (Fig. 3A-D), which both mark myogenic cells in the somites (Comai et al., 2014; Relaix et al., 2005). Thus, both $\mathrm{Pbx} 1$ and $\mathrm{Pbx} 2$ are expressed in embryonic mouse myogenic progenitors. 
Conditional loss of Pbxl and Pbx2 reveals requirements for mouse Pbx proteins in early fast muscle differentiation

Because several studies have shown that $\mathrm{Pbx} 1$ and $\mathrm{Pbx} 2$ have compensatory and overlapping functions (Capellini et al., 2006; Ferretti et al., 2011; Koss et al., 2012), we next wanted to address requirements for both $\mathrm{Pbx} 1$ and $\mathrm{Pbx} 2$ in early mouse muscle differentiation. $P b x 2^{-/-}$mice are viable (Selleri et al., 2004). $P b x 1^{-/} ; P b x 2^{-/}$compound constitutive mutant embryos have multiple organogenesis defects and die by E10.5 (Capellini et al., 2006), precluding an examination of early muscle differentiation. Therefore, we decided to examine conditional loss of $P b x 1$ on a $P b x 2^{-/-}$background (Ferretti et al., 2011; Koss et al., 2012). We used the Myf5-Cre deleter strain (Gensch et al., 2008; Tallquist et al., 2000) in combination with the conditional $P b x l$ allele $\left(P b x I^{f l}\right.$; Koss et al, 2012) to remove Pbx1 from early myogenic cells. In $\mathrm{Myf5}^{\mathrm{Cre} /+} ; \mathrm{Pbx} \mathrm{flfl}^{\mathrm{flfl}} ; \mathrm{Pbx} 2^{-/-}$embryos, we used antibody staining to show loss of Pbx1 in Myf5expressing cells in the somites (Fig. 3E-G), confirming efficient Cre-mediated inactivation of Pbx1. We find that $M y f 5^{C r e /+} ; P b x 1^{f l f l}$ mice are viable and exhibit kyphosis as adults (data not shown), indicating a possible muscle weakness phenotype. We crossed $M y f 5^{\mathrm{Cre} /+} ; \mathrm{Pbx} \mathrm{flfl}^{f l}$ mice with the $\mathrm{Pbx} 2^{-/-}$line to remove both $\mathrm{Pbx} 1$ and $\mathrm{Pbx} 2$ from muscle precursors. In crosses of $P b x 1^{f l f f l} ; P b x 2^{-/-}$mice with $M y f 5^{C r e /+} ; P b x 1^{f l /+} ; P b x 2^{-/-}$mice, we observe expected Mendelian ratios for conditional double mutant $M y f 5^{C r e /+} ; P b x 1^{f l f f l} ; P b x 2^{-/-}$embryos at E11.5 and E17.5, but we obtain negligible numbers of $M y f 5^{C r e /+} ; P b x 1^{f l f f l} ; P b x 2^{-/-}$pups at postnatal day 7 (Table 2). From multiple litters, we were able to identify 3 dead P0-P1 pups in the cage bedding, that, upon genotyping were $\mathrm{Myf5^{Cre/+ }} ; \mathrm{Pbx} 1^{f l / f l} ; \mathrm{Pbx} 2^{-/}$. These results indicate that $\mathrm{Myf5^{ \textrm {Cre } / + }} ; \mathrm{Pb} x \mathrm{I}^{f l / f l} ; \mathrm{Pb} x 2^{-/-}$ mutant animals likely die at or just after birth. 
To determine the effects of conditional loss of $\mathrm{Pbx} 1$ and $\mathrm{Pbx} 2$ on early muscle differentiation, we collected RNA from whole $M y f 5^{C r e /+} ; P b x 1^{f l f l} ; P b x 2^{-/-}$embryos and their sibling controls at E11.5 and performed RNA-seq. Using the cut-offs of 1.2 fold change (FC) and $\mathrm{P}<0.05$, we identified 229 genes down-regulated and 144 genes up-regulated in $\mathrm{Myf5}^{\mathrm{Cre} /+} ; \mathrm{Pbx}{ }^{f l / f l} ; \mathrm{Pbx} 2^{-/-}$mutants (Supplementary Tables S1-S2). DAVID bioinformatics analysis (Huang da et al., 2009) revealed significant enrichment of multiple functional annotation terms related to muscle development and differentiation in the genes down-regulated in $M y f 5^{\mathrm{Cre} /+} ; \mathrm{Pbx} 1^{f l f l} ; \mathrm{Pbx} 2^{-/-}$mutant embryos (Fig. 4A). For the up-regulated gene set, DAVID analysis did not identify any functional annotation terms with significant enrichment following multiple testing correction (not shown). Within the gene set showing downregulation in $\mathrm{Myf5}^{\mathrm{Cre} /+} ; \mathrm{Pbx} \mathrm{I}^{\mathrm{fllfl}} ; \mathrm{Pbx2^{-/ }}$ embryos (Table S1), we noticed the presence of genes associated with fast-twitch muscle differentiation, including genes that we showed to be Pbx-dependent in zebrafish, such as Mylpf (Fig. 1; Maves et al., 2007; Yao et al., 2013). We directly examined muscle fiber-type-associated genes (Schiaffino and Reggiani, 2011) in the RNA-seq data and found 11/13 fast muscle genes with significant down-regulation $(\mathrm{FC}>1.2, \mathrm{P}>0.05)$ in $\mathrm{Myf5}^{\mathrm{Cre} /+} ; \mathrm{Pbx} \mathrm{I}^{\mathrm{flfl}} ; \mathrm{Pbx} 2^{-/-}$embryos, whereas 9/14 slow muscle genes showed either up-regulation or no significant change (Fig. 4B). We used qRT-PCR to validate some of these genes on an

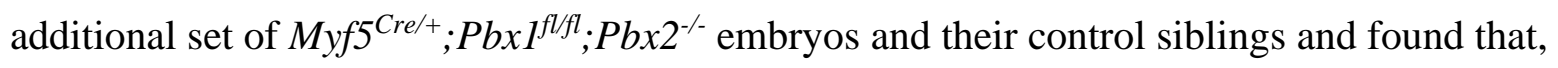
indeed, fast muscle genes showed significant down-regulation, whereas slow muscle genes generally are unaffected (Fig. 4C).

We next validated the results from our RNA-seq and qRT-PCR analyses by performing RNA in situ hybridization experiments. We tested Myog and some of the top down-regulated fast muscle genes (Tnnc2, Myh3, Atp2a1, Myl1, and Mylpf) by RNA in situ at E11.5 (Fig. 5). We 
observe that these fast muscle genes show reduced expression in $\mathrm{Myf}^{\mathrm{Cre} /+} ; \mathrm{Pbx} \mathrm{fllfl}_{\mathrm{fl}}^{\mathrm{Pb}} \mathrm{x} 2^{-/-}$ embryos compared to somite-stage matched control siblings (Fig. 5). Slow muscle genes Myl4, $M y h 7$, and Myh6 appear largely unaffected in $M y f 5^{C r e /+} ; P b x 1^{f l f l} ; P b x 2^{-/-}$embryos at E11.5 (Fig. 5). In addition, $M y h 3, M y l 4, M y h 7$, and $M y h 6$ have strong heart expression that appears unaffected in $\mathrm{Myf}^{\mathrm{Cre} /+} ; \mathrm{Pbx} 1^{f l f l} ; \mathrm{Pbx} 2^{-/-}$embryos (Fig. 5). These findings are consistent with our RNA-seq and qRT-PCR analyses and establish that Pbx proteins are needed for the proper activation of a program of fast muscle differentiation genes in mouse embryogenesis. Taken together, these results confirm that Pbx proteins are needed for the proper activation of a program of fast muscle differentiation genes in both mouse and zebrafish embryogenesis.

\section{Discussion}

Here we show evolutionary conservation of requirements for $\mathrm{Pbx}$ proteins in mammalian and zebrafish embryonic fast-twitch muscle differentiation. These results establish that $\mathrm{Pbx}$ homeodomain proteins have conserved roles in the control of the activation of fast muscle differentiation genes in different species.

Because of the redundancy of, and early requirements for, $\mathrm{Pbx}$ proteins in mouse embryogenesis (reviewed in Selleri et al. 2019), we needed to make use of conditional loss-offunction in $\mathrm{Myf5}^{\mathrm{Cre} /+} ; \mathrm{Pbx} \mathrm{I}^{\mathrm{fl} / \mathrm{fl}} ; \mathrm{Pbx} 2^{-/-}$compound mutants. A similar approach, using different conditional Cre lines, has previously been taken to study $\mathrm{Pbx}$ functions in craniofacial, spleen, and spinal cord motor neuron development and in adult neurogenesis (Ferretti et al., 2011;

Grebbin et al., 2016; Hanley et al., 2016; Koss et al., 2012; Losa et al., 2018; Welsh et al., 2018). Based on their expression patterns, $P b x 1$ and $P b x 2$ are the $P b x$ genes most likely to be involved 
in mouse embryonic skeletal muscle development (Capellini et al., 2006; Di Giacomo et al., 2006; Wagner et al., 2001). Future studies could additionally include loss of Pbx3 (Rhee et al., 2004) to determine whether this Pbx family member also functions in muscle development and fiber-type differentiation.

We chose the Myf5-Cre line to conditionally knock out $P b x l$ in early skeletal muscle development because $M y f 5$ is expressed early in developing somites in the presumptive primary myotome, prior to Myodl expression (Kassar-Duchossoy et al., 2004; Ott et al., 1991). The Myf5-Cre line we employed has been previously used to examine satellite cells in limb and extraocular muscles, to ablate embryonic myogenic cells, and to conditionally ablate genes in skeletal muscle (Comai et al., 2014; Gensch et al., 2008; Greschik et al., 2017; Kuang et al., 2007; Stuelsatz et al., 2014). We note that the Myf5-Cre allele may not be effecting a full deletion of Pbxl in all myoblasts (Fig. 3; Comai et al., 2014), possibly explaining our finding that slow muscle gene $M y h 7$ is upregulated in $P b x 1^{-/-}$mouse embryos (Fig. 2B) but does not appear affected in $\mathrm{Myf5}^{\mathrm{Cre} /+} ; \mathrm{Pbx} \mathrm{flfl}^{f l \mathrm{Pbx}} 2^{-/-}$embryos (Fig. 4C). However, we observe similar defects in the activation of fast muscle differentiation following both conditional inactivation of $P b x 1$ and $P b x 2$ in mouse embryos and whole-animal mzpbx2;pbx4 loss-of-function in zebrafish embryos. These results indicate that the $M y f 5$-Cre line is enabling adequate $P b x$ gene inactivation, leading to a deeper understanding of the function of this family of homeodomain proteins in muscle development in different species.

The defects in activation of fast muscle differentiation genes that we observe in $\mathrm{Myf5}^{\mathrm{Cre} /+} ; \mathrm{Pbx} 1^{\mathrm{flfl} f} ; \mathrm{Pbx2^{-/- }}$ mouse embryos are similar to those observed in Six 1;Six4 double mutant mouse embryos (Niro et al., 2010). In Six $1^{-/-} ;$Six $4^{-/-}$mouse embryos, activation of fast muscle genes, including Atp2a1, Myll, and Tnnc2, is inhibited, while slow muscle genes, such as 
Myl4, are still expressed (Niro et al., 2010). Six1 and Six4 proteins directly bind regulatory regions of fast muscle genes (Niro et al., 2010). In future studies, it will be of interest to examine potential genetic and biochemical interactions between $\mathrm{Pbx}$ proteins and Six proteins during muscle development. In addition, such further studies, using both zebrafish and mice, could help advance our understanding of the conserved and non-conserved roles for Pbx and Six factors in muscle development and fiber-type differentiation during vertebrate evolution.

In conclusion, we provide evidence for evolutionarily conserved regulation of embryonic fast muscle differentiation by Pbx factors. In future studies, we plan to determine the requirements for Pbx factors in later stages of muscle development and fiber-type differentiation in both zebrafish and mice. In addition, we plan to take advantage of our zebrafish and mouse mutant strains to examine, in developing embryos, the Pbx-dependent chromatin events in myogenesis and muscle differentiation. The goal will be to determine whether Pbx proteins are needed during skeletal muscle development to open chromatin, or to promote Myod1 binding or Myod1 activity. Ultimately, these studies will help us understand how broadly-expressed factors like Pbx help master regulatory factors like Myod1 activate their transcriptional targets in a temporally and spatially controlled manner to drive cell-type-specific differentiation programs in different species.

\section{Acknowledgements}

We thank Peter Currie, Zhiyuan Gong, Phil Ingham, Heike Pöpperl, and Phil Soriano for generously providing reagents. The Zebrafish International Resource Center (supported by grants RR12546 and RR15402-01 from the NIH) provided cDNA clones. We thank the FHCRC 
Genomics Shared Resource for excellent technical assistance with the RNA-seq experiments. We thank the SCRI Aquatics Facility for zebrafish care. This work was supported by NIH

1R03AR057477 (L.M.), funded in part by the American Recovery and Reinvestment Act, NIH 1R03AR065760 (L.M.), the Seattle Children's Myocardial Regeneration Initiative, by NIH 1R01AR45113 (S.J.T.), and by NIH RO1 DE024745 (L.S.).

\section{References}

Bassel-Duby, R., Olson, E.N., 2006. Signaling pathways in skeletal muscle remodeling. Annu. Rev. Biochem. 75, 19-37.

Berkes, C.A., Bergstrom, D.A., Penn, B.H., Seaver, K.J., Knoepfler, P.S., Tapscott, S.J., 2004. $\mathrm{Pbx}$ marks genes for activation by $\mathrm{MyoD}$ indicating a role for a homeodomain protein in establishing myogenic potential. Mol. Cell 14, 465-477.

Berkes, C.A., Tapscott, S.J., 2005. MyoD and the transcriptional control of myogenesis. Semin. Cell Dev. Biol. 16, 585-595.

Bessarab, D.A., Chong, S.W., Srinivas, B.P., Korzh, V., 2008. Six1a is required for the onset of fast muscle differentiation in zebrafish. Dev. Biol. 323, 216-228.

Biressi, S., Molinaro, M., Cossu, G., 2007. Cellular heterogeneity during vertebrate skeletal muscle development. Dev. Biol. 308, 281-293.

Blaauw, B., Schiaffino, S., Reggiani, C., 2013. Mechanisms modulating skeletal muscle phenotype. Compr. Physiol. 3, 1645-1687.

Blais, A., Tsikitis, M., Acosta-Alvear, D., Sharan, R., Kluger, Y., Dynlacht, B.D., 2005. An initial blueprint for myogenic differentiation. Genes Dev. 19, 553-569. 
Buckingham, M., Vincent, S.D., 2009. Distinct and dynamic myogenic populations in the vertebrate embryo. Curr. Opin. Genet. Dev. 19, 444-453.

Cao, Y., Kumar, R.M., Penn, B.H., Berkes, C.A., Kooperberg, C., Boyer, L.A., Young, R.A., Tapscott, S.J., 2006. Global and gene-specific analyses show distinct roles for Myod and Myog at a common set of promoters. EMBO J. 25, 502-511.

Cao, Y., Yao, Z., Sarkar, D., Lawrence, M., Sanchez, G.J., Parker, M.H., MacQuarrie, K.L., Davison, J., Morgan, M.T., Ruzzo, W.L., Gentleman, R.C., Tapscott, S.J., 2010. Genomewide MyoD binding in skeletal muscle cells: A potential for broad cellular reprogramming. Dev. Cell 18, 662-674.

Capellini, T.D., Di Giacomo, G., Salsi, V., Brendolan, A., Ferretti, E., Srivastava, D., Zappavigna, V., Selleri, L., 2006. Pbx1/Pbx2 requirement for distal limb patterning is mediated by the hierarchical control of Hox gene spatial distribution and Shh expression. Development 133, 2263-2273.

Cho, O.H., Mallappa, C., Hernández-Hernández, J.M., Rivera-Pérez, J.A., Imbalzano, A.N., 2015. Contrasting roles for MyoD in organizing myogenic promoter structures during embryonic skeletal muscle development. Dev. Dyn. 244, 43-55.

Ciciliot, S., Rossi, A.C., Dyar, K.A., Blaauw, B., Schiaffino, S., 2013. Muscle type and fiber type specificity in muscle wasting. Int. J. Biochem. Cell. Biol. 45, 2191-2199.

Comai, G., Sambasivan, R., Gopalakrishnan, S., Tajbakhsh, S., 2014. Variations in the efficiency of lineage marking and ablation confound distinctions between myogenic cell populations. Dev Cell 31, 654-667. 
de la Serna, I.L., Ohkawa, Y., Berkes, C.A., Bergstrom, D.A., Dacwag, C.S., Tapscott, S.J., Imbalzano, A.N., 2005. MyoD targets chromatin remodeling complexes to the myogenin locus prior to forming a stable DNA-bound complex. Mol. Cell. Biol. 25, 3997-4009.

Dell'Orso, S., Wang, A.H., Shih, H.Y., Saso, K., Berghella, L., Gutierrez-Cruz, G., Ladurner, A.G., O'Shea, J.J., Sartorelli, V., Zare, H., 2016. The Histone Variant MacroH2A1.2 Is Necessary for the Activation of Muscle Enhancers and Recruitment of the Transcription Factor Pbx1. Cell Rep. 14, 1156-1168.

Di Giacomo, G., Koss, M., Capellini, T.D., Brendolan, A., Pöpperl, H., Selleri, L., 2006. Spatiotemporal expression of $\mathrm{Pbx} 3$ during mouse organogenesis. Gene Expr. Patterns 6, 747-757.

Ekmark, M., Rana, Z.A., Stewart, G., Hardie, D.G., Gundersen, K., 2007. De-phosphorylation of MyoD is linking nerve-evoked activity to fast myosin heavy chain expression in rodent adult skeletal muscle: MyoD and muscle fibre type. J. Physiol. 584, 637-650.

Farr, G.H. 3rd, Imani, K., Pouv, D., Maves L., 2018. Functional testing of a human PBX3 variant in zebrafish reveals a potential modifier role in congenital heart defects. Dis. Model. Mech. 11.

Ferretti, E., Li, B., Zewdu, R., Wells, V., Hebert, J.M., Karner, C., Anderson, M.J., Williams, T., Dixon, J., Dixon, M.J., Depew, M.J., Selleri. L. 2011. A conserved Pbx-Wnt-p63-Irf6 regulatory module controls face morphogenesis by promoting epithelial apoptosis. Dev. Cell 21, 627-641.

Fong, A.P., Tapscott, S.J., 2013. Skeletal muscle programming and re-programming. Curr. Opin. Genet. Dev. 23, 568-573. 
Gensch, N., Borchardt, T., Schneider, A., Riethmacher, D., Braun, T., 2008. Different autonomous myogenic cell populations revealed by ablation of Myf5-expressing cells during mouse embryogenesis. Development 135, 1597-1604.

Grebbin, B.M., Hau, A.C., Groß, A., Anders-Maurer, M., Schramm, J., Koss, M., Wille, C., Mittelbronn, M., Selleri, L., Schulte, D., 2016. Pbx1 is required for adult subventricular zone neurogenesis. Development 143, 2281-2291.

Grebbin, B.M., Schulte, D., 2017. PBX1 as Pioneer Factor: A Case Still Open. Front Cell Dev Biol. 5, 9.

Greschik, H., Duteil, D., Messaddeq, N., Willmann, D., Arrigoni, L., Sum, M., Jung, M., Metzger, D., Manke, T., Günther, T., Schüle, R., 2017. The histone code reader Spin1 controls skeletal muscle development. Cell Death Dis. 8, e3173.

Grifone, R., Demignon, J., Houbron, C., Souil, E., Niro, C., Seller, M.J., Hamard, G., Maire, P., 2005. Six 1 and Six4 homeoproteins are required for Pax3 and Mrf expression during myogenesis in the mouse embryo. Development 132, 2235-2249.

Hagiwara, N., Ma, B., Ly, A., 2005. Slow and fast fiber isoform gene expression is systematically altered in skeletal muscle of the Sox6 mutant, p100H. Dev. Dyn. 234, 301311.

Hagiwara, N., Yeh, M., Liu, A., 2007. Sox6 is required for normal fiber type differentiation of fetal skeletal muscle in mice. Dev. Dyn. 236, 2062-2076.

Hammond, C.L., Hinits, Y., Osborn, D.P., Minchin, J.E., Tettamanti, G., Hughes, S.M., 2007. Signals and myogenic regulatory factors restrict pax 3 and pax 7 expression to dermomyotome-like tissue in zebrafish. Dev. Biol. 302, 504-521. 
Hanley, O., Zewdu, R., Cohen, L.J., Jung, H., Lacombe, J., Philippidou, P., Lee, D.H., Selleri, L., Dasen, J.S., 2016. Parallel Pbx-Dependent Pathways Govern the Coalescence and Fate of Motor Columns. Neuron 91, 1005-1020.

Heidt, A.B., Rojas, A., Harris, I.S., Black, B.L., 2007. Determinants of myogenic specificity within MyoD are required for noncanonical E box binding. Mol. Cell. Biol. 27, 5910-5920.

Hernández-Hernández, J.M., García-González, E.G., Brun, C.E., Rudnicki, M.A., 2017. The myogenic regulatory factors, determinants of muscle development, cell identity and regeneration. Semin. Cell Dev. Biol. 72, 10-18.

Hinits, Y., Williams, V.C., Sweetman, D., Donn, T.M., Ma, T.P., Moens, C.B., Hughes, S.M., 2011. Defective cranial skeletal development, larval lethality and haploinsufficiency in Myod mutant zebrafish. Dev. Biol. 358, 102-112.

Huang da, W., Sherman, B.T., Lempicki, R.A., 2009. Systematic and integrative analysis of large gene lists using DAVID bioinformatics resources. Nat. Protoc. 4, 44-57.

Hughes, S.M., Kioshi, K., Rudnicki, M.A., Maggs, A.M., 1997. MyoD protein is differentially accumulated in fast and slow skeletal muscle fibres and required for normal fibre type balance in rodents. Mech. Dev. 61, 151-163.

Hwang, W.Y., Fu, Y., Reyon, D., Maeder, M.L., Tsai, S.Q., Sander, J.D., Peterson, R.T., Yeh, J.R. J., Joung, J.K., 2013. Efficient genome editing in zebrafish using a CRISPR-Cas system. Nat. Biotech. 31, 227-229.

Jackson, H.E., Ingham, P.W., 2013. Control of muscle fibre-type diversity during embryonic development: The zebrafish paradigm. Mech. Dev. 130, 447-457.

Jackson, H.E., Ono, Y., Wang, X., Elworthy, S., Cunliffe, V.T., Ingham, P.W., 2015. The role of Sox6 in zebrafish muscle fiber type specification. Skelet. Muscle 5, 2. 
Jacobs, Y., Schnabel, C.A., Cleary, M.L., 1999. Trimeric association of Hox and TALE homeodomain proteins mediates Hoxb2 hindbrain enhancer activity. Mol. Cell. Biol. 19, 5134-5142.

Kassar-Duchossoy, L., Gayraud-Morel, B., Gomès, D., Rocancourt, D., Buckingham, M., Shinin, V., Tajbakhsh, S., 2004. Mrf4 determines skeletal muscle identity in Myf5:Myod doublemutant mice. Nature 431, 466-471.

Koss, M., Bolze, A., Brendolan, A., Saggese, M., Capellini, T.D., Bojilova, E., Boisson, B., Prall, O.W., Elliott, D.A., Solloway, M., Lenti, E., Hidaka, C., Chang, C.P., Mahlaoui, N., Harvey, R.P., Casanova, J.L., Selleri, L., 2012. Congenital asplenia in mice and humans with mutations in a Pbx/Nkx2-5/p15 module. Dev. Cell 22, 627-641.

Kuang, S., Kuroda, K., Le Grand, F., Rudnicki, M.A., 2007. Asymmetric self-renewal and commitment of satellite stem cells in muscle. Cell 129, 999-1010.

Lassar, A.B., 2017. Finding MyoD and lessons learned along the way. Semin Cell Dev Biol. 72, 3-9.

Li, H., Handsaker, B., Wysoker, A., Fennell, T., Ruan, J., Homer, N., Marth, G., Abecasis, G., Durbin, R., 1000 Genome Project Data processing Subgroup, 2009. The Sequence Alignment/Map format and SAMtools. Bioinformatics 25, 2078-2079.

Ljubicic, V., Burt, M., Jasmin, B.J., 2014. The therapeutic potential of skeletal muscle plasticity in Duchenne muscular dystrophy: phenotypic modifiers as pharmacologic targets. FASEB J. $28,548-568$.

Losa, M., Risolino, M., Li, B., Hart, J., Quintana, L., Grishina, I., Yang, H., Choi, I.F., Lewicki, P., Khan, S., Aho, R., Feenstra, J., Vincent, C.T., Brown, A.M.C., Ferretti, E., Williams, T., 
Selleri, L., 2018. Face morphogenesis is promoted by Pbx-dependent EMT via regulation of Snail1 during frontonasal prominence fusion. Development 145.

Macharia, R., Otto, A., Valasek, P., Patel, K., 2010. Neuromuscular junction morphology, fibertype proportions, and satellite-cell proliferation rates are altered in MyoD-/- mice. Muscle Nerve 42, 38-52.

Maves, L., Waskiewicz, A.J., Paul, B., Cao, Y., Tyler, A., Moens, C.B., Tapscott, S.J., 2007. Pbx homeodomain proteins direct Myod activity to promote fast-muscle differentiation. Development 134, 3371-3382.

Moens, C.B., Selleri, L., 2006. Hox cofactors in vertebrate development. Dev. Biol. 291, 193206.

Neff, M.M., Neff, J.D., Chory, J., Pepper A.E., 1998. dCAPS, a simple technique for the genetic analysis of single nucleotide polymorphisms: experimental applications in Arabidopsis thalania genetics. Plant J. 14, 387-392.

Niro, C., Demignon, J., Vincent, S., Liu, Y., Giordani, J., Sgarioto, N., Favier, M., GuilletDeniau, I., Blais, A., Maire, P., 2010. Six 1 and Six4 gene expression is necessary to activate the fast-type muscle gene program in the mouse primary myotome. Dev. Biol. 338, 168-182.

Nord, H., Nygård Skalman, L., von Hofsten, J., 2013. Six1 regulates proliferation of Pax7positive muscle progenitors in zebrafish. J. Cell Sci. 126, 1868-1880.

O'Brien, J.H., Hernandez-Lagunas, L., Artinger, K.B., Ford, H.L., 2014. MicroRNA-30a regulates zebrafish myogenesis through targeting the transcription factor Six1. J. Cell Sci. $127,2291-2301$.

Ott, M.O., Bober, E., Lyons, G., Arnold, H., Buckingham, M., 1991. Early expression of the myogenic regulatory gene, myf-5, in precursor cells of skeletal muscle in the mouse embryo. 
Development 111, 1097-1107.

Pöpperl, H., Rikhof, H., Chang, H., Haffter, P., Kimmel, C.B., Moens, C.B., 2000. lazarus is a novel pbx gene that globally mediates hox gene function in zebrafish. Mol Cell 6, 255-267.

Pliner, H.A., Packer, J.S., McFaline-Figueroa, J.L., Cusanovich, D.A., Daza, R.M., Aghamirzaie, D., Srivatsan, S., Qiu, X., Jackson, D., Minkina, A., Adey, A.C., Steemers, F.J., Shendure, J., Trapnell, C., 2018. Cicero Predicts cis-Regulatory DNA Interactions from Single-Cell Chromatin Accessibility Data. Mol. Cell. 71, 858-871.

Relaix, F., Rocancourt, D., Mansouri, A., Buckingham, M., 2005. A Pax3/Pax7-dependent population of skeletal muscle progenitor cells. Nature 435, 948-953.

Rhee, J.W., Arata, A., Selleri, L., Jacobs, Y., Arata, S., Onimaru, H., Cleary, M.L., 2004. Pbx3 deficiency results in central hypoventilation. Am. J. Pathol. 165, 1343-1350.

Richard, A.F., Demignon, J., Sakakibara, I., Pujol, J., Favier, M., Strochlic, L., Le Grand, F., Sgarioto, N., Guernec, A., Schmitt, A., Cagnard, N., Huang, R., Legay, C., Guillet-Deniau, I., Maire, P., 2011. Genesis of muscle fiber-type diversity during mouse embryogenesis relies on Six1 and Six4 gene expression. Dev. Biol. 359, 303-320.

Rossi, G., Messina, G., 2014. Comparative myogenesis in teleosts and mammals. Cell. Mol. Life Sci. 71, 3081-3099.

Ruzicka, L., Howe, D.G., Ramachandran, S., Toro, S., Van Slyke, C.E., Bradford, Y.M., Eagle, A., Fashena, D., Frazer, K., Kalita, P., Mani, P., Martin, R., Moxon, S.T., Paddock, H., Pich, C., Schaper, K., Shao, X., Singer, A., Westerfield, M., 2019. The Zebrafish Information Network: new support for non-coding genes, richer Gene Ontology annotations and the Alliance of Genome Resources. Nucleic Acids Res. 47, D867-D873.

Schiaffino, S., Reggiani, C., 2011. Fiber Types in Mammalian Skeletal Muscles. Physiol. Rev. 
91, 1447-1531.

Selleri, L., Depew, M.J., Jacobs, Y., Chanda, S.K., Tsang, K.Y., Cheah, K.S., Rubenstein, J.L., O’Gorman, S., Cleary, M.L., 2001. Requirement for Pbx1 in skeletal patterning and programming chondrocyte proliferation and differentiation. Development 128, 3543-3557.

Selleri, L., DiMartino, J., van Deursen, J., Brendolan, A., Sanyal, M., Boon, E., Capellini, T., Smith, K.S., Rhee, J., Pöpperl, H., Grosveld, G., Cleary, M.L., 2004. The TALE homeodomain protein $\mathrm{Pbx} 2$ is not essential for development and long-term survival. Mol. Cell. Biol. 24, 5324-5331.

Selleri, L., Zappavigna, V., Ferretti, E., 2019. 'Building a perfect body': control of vertebrate organogenesis by PBX-dependent regulatory networks. Genes Dev. 33, 258-275.

Seward, D.J., Haney, J.C., Rudnicki, M.A., Swoap, S.J., 2001. bHLH transcription factor MyoD affects myosin heavy chain expression pattern in a muscle-specific fashion. Am. J. Physiol. Cell Physiol. 280, C408-C413.

Soleimani, V.D., Yin, H., Jahani-Asl, A., Ming, H., Kockx, C.E., van Ijcken, W.F., Grosveld, F., Rudnicki, M.A., 2012. Snail regulates MyoD binding-site occupancy to direct enhancer switching and differentiation-specific transcription in myogenesis. Mol. Cell 47, 457-468.

Stuelsatz, P., Shearer, A., Yablonka-Reuveni, Z., 2014. Ancestral Myf5 gene activity in periocular connective tissue identifies a subset of fibro/adipogenic progenitors but does not connote a myogenic origin. Dev. Biol. 385, 366-379.

Talbot, J., Maves, L., 2016. Skeletal muscle fiber type: using insights from developmental biology to dissect targets for susceptibility and resistance to muscle disease. Wiley Interdiscip. Rev. Dev. Biol. 5, 518-534.

Talbot, J.C., Teets, E.M., Ratnayake, D., Duy, P.Q., Currie, P.D., Amacher, S.L., 2019. Muscle 
precursor cell movements in zebrafish are dynamic and require Six family genes.

Development 146(10).

Tallquist, M.D., Weismann, K.E., Hellström, M., Soriano, P., 2000. Early myotome specification regulates PDGFA expression and axial skeletal development. Development 127, 5059-5070.

Trapnell, C., Pachter, L., Salzberg, S., 2009. TopHat: discovering splice junctions with RNASeq. Bioinformatics 25, 1105-1111.

Vincent, S.D., Mayeuf, A., Niro, C., Saitou, M., Buckingham, M., 2012. Non conservation of function for the evolutionarily conserved prdm 1 protein in the control of the slow twitch myogenic program in the mouse embryo. Mol. Biol. Evol. 29, 3181-3191.

von Hofsten, J., Elworthy, S., Gilchrist, M.J., Smith, J.C., Wardle, F.C., Ingham, P.W., 2008. Prdm1- and Sox6-mediated transcriptional repression specifies muscle fibre type in the zebrafish embryo. EMBO Rep. 9, 683-689.

Wagner, K., Mincheva, A., Korn, B., Lichter, P., Pöpperl, H., 2001. Pbx4, a new Pbx family member on mouse chromosome 8, is expressed during spermatogenesis. Mech. Dev. 103, $127-131$.

Waskiewicz, A.J., Rikhof, H.A., Moens, C.B., 2002. Eliminating zebrafish pbx proteins reveals a hindbrain ground state. Dev. Cell 3, 723-733.

Welsh, I.C., Hart, J., Brown, J.M., Hansen, K., Rocha Marques, M., Aho, R.J., Grishina, I., Hurtado, R., Herzlinger, D., Ferretti, E., Garcia-Garcia, M.J., Selleri, L., 2018. Pbx loss in cranial neural crest, unlike in epithelium, results in cleft palate only and a broader midface. J. Anat. 233. 222-242.

Westerfield, M., 2000. The zebrafish book. A guide for the laboratory use of zebrafish (Danio rerio). $4^{\text {th }}$ ed., University of Oregon Press, Eugene. 
Yao, Z., Farr, G.H. 3rd, Tapscott, S.J., Maves, L., 2013. Pbx and Prdm1a transcription factors differentially regulate subsets of the fast skeletal muscle program in zebrafish. Biol. Open 2, $546-555$.

Zammit, P.S., 2017.Function of the myogenic regulatory factors Myf5, MyoD, Myogenin and MRF4 in skeletal muscle, satellite cells and regenerative myogenesis. Semin. Cell Dev. Biol. 72, 19-32. 
Tables

\begin{tabular}{|c|c|}
\hline $\begin{array}{l}\text { Gene } \\
\text { (Z-zebrafish; } \\
\text { M-mouse) }\end{array}$ & Primer Sequence (Forward 5'-3'; Reverse 5'-3') \\
\hline \multicolumn{2}{|c|}{ Animal genotyping } \\
\hline$p b \times 2^{s c m 10}(Z)$ & $\begin{array}{l}\text { CCATGAGAAATGTTCAGGAGGAG; } \\
\text { TCGCAGCAGATACTGCCGCTGCGGCTCCGCCACCTTT }\end{array}$ \\
\hline$p b \times 4^{b 557}(Z)$ & ACTCGGCGGACTCTCGCAAGC; GGCTCTCGTCGGTGATGGCCATGATCT \\
\hline$P b \times 1(\mathrm{M})$ & TGAGTATTCGGGGAGCCCAAGAA; CATGATGCCCAGTCTGTAGGGGT \\
\hline$P b x 1$ neo $(\mathrm{M})$ & CTATCAGGACATAGCGTTGG \\
\hline$P b x 1^{f l}(\mathrm{M})$ & ATCAGTGCCATTTCTCCAGCC; TGGGTATCCATTCCAAAGGTGG \\
\hline$P b \times 2(\mathrm{M})$ & TCCTTAAGTCTGTTTGCCTCGCTG; CTGCTCATACTTCTCCAGCTCTGA \\
\hline$P b \times 2$ neo $(\mathrm{M})$ & CTATCAGGACATAGCGTTGG \\
\hline Myf5 Cre (M) & CGTAGACGCCTGAAGAAGGTCAAC; CACATTAGAAAACCTGCCAACACC \\
\hline Myf5Cre lox (M) & ACGAAGTTATTAGGTCCCTCGAC \\
\hline \multicolumn{2}{|l|}{$\begin{array}{l}\text { RNA in situ } \\
\text { probes }\end{array}$} \\
\hline Tnnc2 (M) & CAACCATGACGGACCAACAG; TCCAGTCACAGAGCCCAAAG \\
\hline $\operatorname{Atp} 2 a 1(\mathrm{M})$ & TGGTGTTGTGGGCATGTTGG; ACACGGTTCAAAGACATGGAGG \\
\hline Myh6 (M) & AGCTGGAGAAGATCCGCAAA; AGAGGTTATTCCTCGTCGTGC \\
\hline Myh3 (M) & AGCCACTGGGGACCTTGCCA; GCAGGCTGCCAGGTCCATCC \\
\hline Myl1 (M) & GGGTCCACCCTCCAGACGCT; GCCAGTCTTCCCCAACATTGCGT \\
\hline Mylpf (M) & GGAGATCTAAGACATGGCAC; GTTTTATTGAGGGCCAGGGT \\
\hline Myl4 (M) & GTCCCACCTCCACTGGAGAGC; AGCTGGGGCTCTTTATTTCC \\
\hline $\operatorname{Myh7}(\mathrm{M})$ & CAGGCCCAGTCCCGAGGTGT; TCCCGGGACAGGTCAGAGCG \\
\hline \multicolumn{2}{|r|}{ 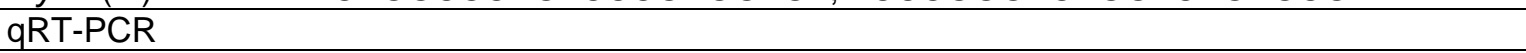 } \\
\hline Odc1 (M) & TTGCCACTGATGATTCCAAA; CATGGAAGCTCACACCAATG \\
\hline$P b \times 1(\mathrm{M})$ & CTCATGCGACTGGACAACAT; TCGGAATGCTCCACTGAGTT \\
\hline $\operatorname{Myog}(\mathrm{M})$ & ATCTCCGCTACAGAGGCGGG; GGTCAGCCGCGAGCAAATGA \\
\hline Myh3 (M) & GGATGCCTGCAGGGACGCAA; GCGCTGGTGTCCTGCTCCTT \\
\hline Mylpf (M) & GGCTGCCGGGGCAGGACTAT; CGCTCCTGCCCTTCTCTTGGC \\
\hline Myl1 (M) & GCAACAGGAGGACTTCAAGGAGGC; CCCGGAGGACGTCTCCCACC \\
\hline Myl4 (M) & AGCGAGGCAGAGGTGGAGCA; TCTCACGCAGGGCCAAGCAC \\
\hline Myh7 (M) & GAGCAAGGCCGAGGAGACGC; TGCCGGGACAGCTCCCCATT \\
\hline Tnnc2 (M) & GGAGTGCGGAGGAGACAACC; ACCGCCATCAGCATCGAACA \\
\hline Atp2a1 (M) & CCAATGAGCTCCCTGCTGAGG; GCAGTGACGGTTTCTTCGCC \\
\hline
\end{tabular}


Table 2 Offspring of $P b \times 1^{f / / f /} ; P b \times 2^{-/-} \times M y f 5^{C r e /+} ; P b \times 1^{f / /+} ; P b \times 2^{-/ \text {Mice }}$

\begin{tabular}{|c|c|c|c|c|c|c|c|c|c|}
\hline \multirow[b]{2}{*}{ Genotype } & \multicolumn{3}{|c|}{ Frequencies at E11.5 } & \multicolumn{3}{|c|}{ Frequencies at E17.5 } & \multicolumn{3}{|c|}{ Frequencies at P7 } \\
\hline & Observed & Expected & $P$ & Observed & Expected & $P$ & Observed & Expected & $\mathrm{P}$ \\
\hline $\begin{array}{l}\text { Myf5Cre/+; } \\
\text { Pbx1fl/fl;Pbx2-/- }\end{array}$ & 25 & 22.25 & 0.902 & 7 & 12 & 0.227 & 2 & 20.25 & $\begin{array}{l}4.335 \\
E-05\end{array}$ \\
\hline $\begin{array}{l}\text { Myf5Cre/+; } \\
\text { Pbx1fl/+;Pbx2-/- }\end{array}$ & 20 & 22.25 & & 13 & 12 & & 29 & 20.25 & \\
\hline$P b \times 1 f l / f l ; P b \times 2-/-$ & 22 & 22.25 & & 17 & 12 & & 27 & 20.25 & \\
\hline$P b \times 1 f l /+; P b \times 2-/-$ & 22 & 22.25 & & 11 & 12 & & 23 & 20.25 & \\
\hline
\end{tabular}

$P$ values calculated using a Chi-square test. 


\section{Figure Legends}

Fig. 1. Fast muscle differentiation genes are downregulated in $m z p b x 2^{-/-} ; p b x 4^{-/-}$zebrafish embryos relative to controls at 18 somite stage/18 hours post fertilization (hpf). (A-D) Whole mount RNA in situ hybridizations in 18 hpf embryos. Top panels of each set show control $p b x 2^{+/+} ; p b x 4^{+/+}$embryos. Bottom panels of each set show somite-stage matched $m z p b x 2^{-/} ; p b x 4^{-}$

/- embryos. Lateral views show anterior to the left and dorsal at the top. $\mathrm{N}=10-16$ control and 3-5 mutant embryos for each condition. (A) Arrow in A points to reduced myog expression in $m z p b x 2^{-/-} ; p b x 4^{-/}$embryo. (B-D) atp $2 a 1, m y l 1$, and $m y l p f a$ appear generally reduced in $m z p b x 2^{-/}$ $; p b \times 4^{-/}$embryos. krox20 is expressed in hindbrain rhombomeres 3 and $5(\mathrm{r} 3, \mathrm{r} 5$ in $\mathrm{A})$ and is downregulated in $m z p b x 2^{-/} ; p b x 4^{-/}$embryos. smyhc1 expression in the developing heart (arrowhead in A) is downregulated in $m z p b x 2^{-/-} ; p b x 4^{-/-}$embryos.

Fig. 2. Loss of Pbx1 has little effect on early mouse muscle differentiation. (A-B) qRT-PCR analysis of muscle gene expression in (A) E9.5 and (B) E11.5 $\mathrm{Pbxl}^{\text {/- }}$ embryos relative to control $P b x 1^{+/+}$embryos. Myh3, Mylpf, and Myll are fast muscle genes. Myl4 and Myh7 are slow muscle genes. $P b x l$ expression is included as a control gene. Expression levels are normalized to the gene $O d c 1$. For each gene, the expression level in $P b x 1^{+/+}$embryos is set to 1 (dashed line in graphs). Box plots show the distribution of the normalized expression level $\left(2^{-\Delta \Delta \mathrm{Ct}}\right)$ of four control/mutant sibling pairs for each gene. The boxes extend from the 25 th to 75 th percentiles, the whiskers are at the minimum and maximum, the bar within the box represents the median, and the cross represents the mean. Statistics are described in Materials and Methods. * $\mathrm{P}<0.02$, 
** $\mathrm{P}<0.004, * * * \mathrm{P}<0.0008, * * * * \mathrm{P}<0.0001$. (C-D) Whole mount Myog RNA in situ hybridization in (C) $P b x 1^{+/+}$and (D) $P b x 1^{-/-}$E11.5 embryos. N=3.

Fig. 3. Pbx1 and Pbx2 expression in mouse embryo somites. (A-B) E11.5 mouse embryo whole mount stained with anti-Pbx1b and anti-Myf5. A, low magnification, scale bar=20 $\mu \mathrm{m}$. B, high magnification of A, scale bar=20 $\mu \mathrm{m}$. (C-D) E11.5 mouse embryo sagittal section stained for anti-Pbx2 and anti-Myod1. C, low magnification, scale bar=10 $\mu \mathrm{m} . \mathrm{D}$, high magnification of $\mathrm{C}$, scale bar=10 $\mu \mathrm{m}$. (E-F) E11.5 Myf5 ${ }^{\mathrm{Cre} /+} ; P b x 1^{f l f l}$ embryo stained with anti-Pbx1b and anti-Myf5. E, low magnification, scale bar as in A. F, high magnification of E, scale bar as in B. Rostral is to the left. (G) Quantification of Myf5 and Pbx1b co-expressing cells (Myf5+Pbx1+) in E11.5 control embryos and $M y f 5^{C r e /+} ; P b x 1^{f l f l} ; P b x 2^{-/-}$mutant embryos.

Fig. 4. $P b x 1$ and $P b x 2$ are needed for mouse embryonic fast muscle gene expression. (A) Functional annotation terms associated with genes downregulated (Table S1) in E11.5

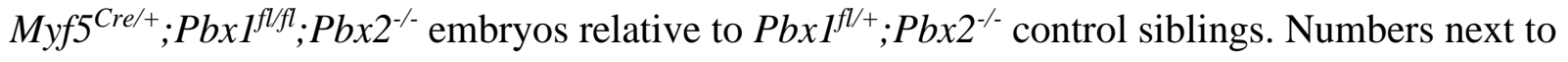
each bar indicate number of genes associated with each term. (B) Muscle fiber type-associated gene expression from RNA-seq in mutant $M y f 5^{C r e /+} ; \mathrm{Pbxl}^{f l f l} ; \mathrm{Pbx2^{-/- }}$ E11.5 embryos relative to $P b x I^{f l /+} ; P b x 2^{-/-}$control siblings. Genes showing significant changes are in bold. Color bar legend illustrates range of fold changes. (C) qRT-PCR analysis of muscle gene expression in E11.5

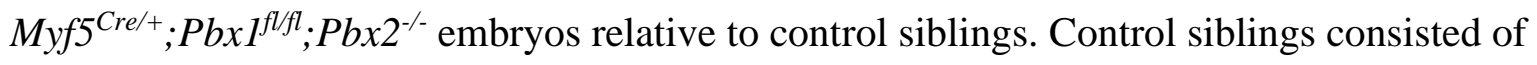
$P b x 1^{f l /+} ; P b x 2^{-/-}(\mathrm{N}=1)$ or $M y f 5^{C r e /+} ; P b x 1^{f l /+} ; P b x 2^{-/-}(\mathrm{N}=4)$ embryos. Tnnc2, Myh3, Atp2al, Myll and Mylpf are fast muscle genes. Myl4 and Myh7 are slow muscle genes. Expression levels are normalized to the gene $O d c 1$. For each gene, the expression level in control sibling embryos is 
set to 1 (dashed line). Box plots show the distribution of the normalized expression level $\left(2^{-\Delta \Delta \mathrm{Ct}}\right)$ of five control/mutant sibling pairs for each gene. The boxes extend from the 25 th to 75 th percentiles, the whiskers are at the minimum and maximum, the bar within the box represents the median, and the cross represents the mean. Statistics are described in Materials and Methods. * $\mathrm{P}<0.05, * * \mathrm{P}<0.01, * * * \mathrm{P}<0.003$.

Fig. 5. Fast muscle differentiation genes are downregulated in $M y f 5^{\mathrm{Cre} /+} ; \mathrm{Pbx} \mathrm{I}^{f l f f l} ; \mathrm{Pbx} 2^{-/-}$embryos relative to control siblings at E11.5. (A-I) Whole mount RNA in situ hybridizations in E11.5 embryos. Left column of each panel shows control embryos, and right column of each panel shows somite-stage matched $\mathrm{Myf5}^{\mathrm{Cre} /+} ; \mathrm{Pbx} \mathrm{I}^{f l f l} ; \mathrm{Pbx} 2^{-/-}$embryos. The pictured control embryos for Myog, Tnnc2, Myh3, Mylpf, Myl4, Myh7, and Myh6 are Myf5 $5^{\mathrm{Cre} /+} ; P b x 1^{f l /+} ; P b x 2^{-/}$. The pictured control embryo for Atp2al is $P b x 1^{f l f f l} ; P b x 2^{-/-}$, and the control for $M y l 1$ is $P b x 1^{f l /+} ; P b x 2^{-/-}$ . No differences in gene expression were noted among controls of different genotypes. Insets show $2 \mathrm{X}$ zoom views at the level of somite 17 (* in each panel). N=3-5 control and 3-5 mutant embryos for each condition. Myh3, Myl4, Myh7, and Myh6 have strong heart expression (h in C) that appears unaffected in $M y f 5^{\mathrm{Cre} /+} ; \mathrm{Pbx} \mathrm{I}^{\mathrm{fl} f \mathrm{fl}} ; \mathrm{Pb} \times 2^{-/-}$embryos. 
Figure 1
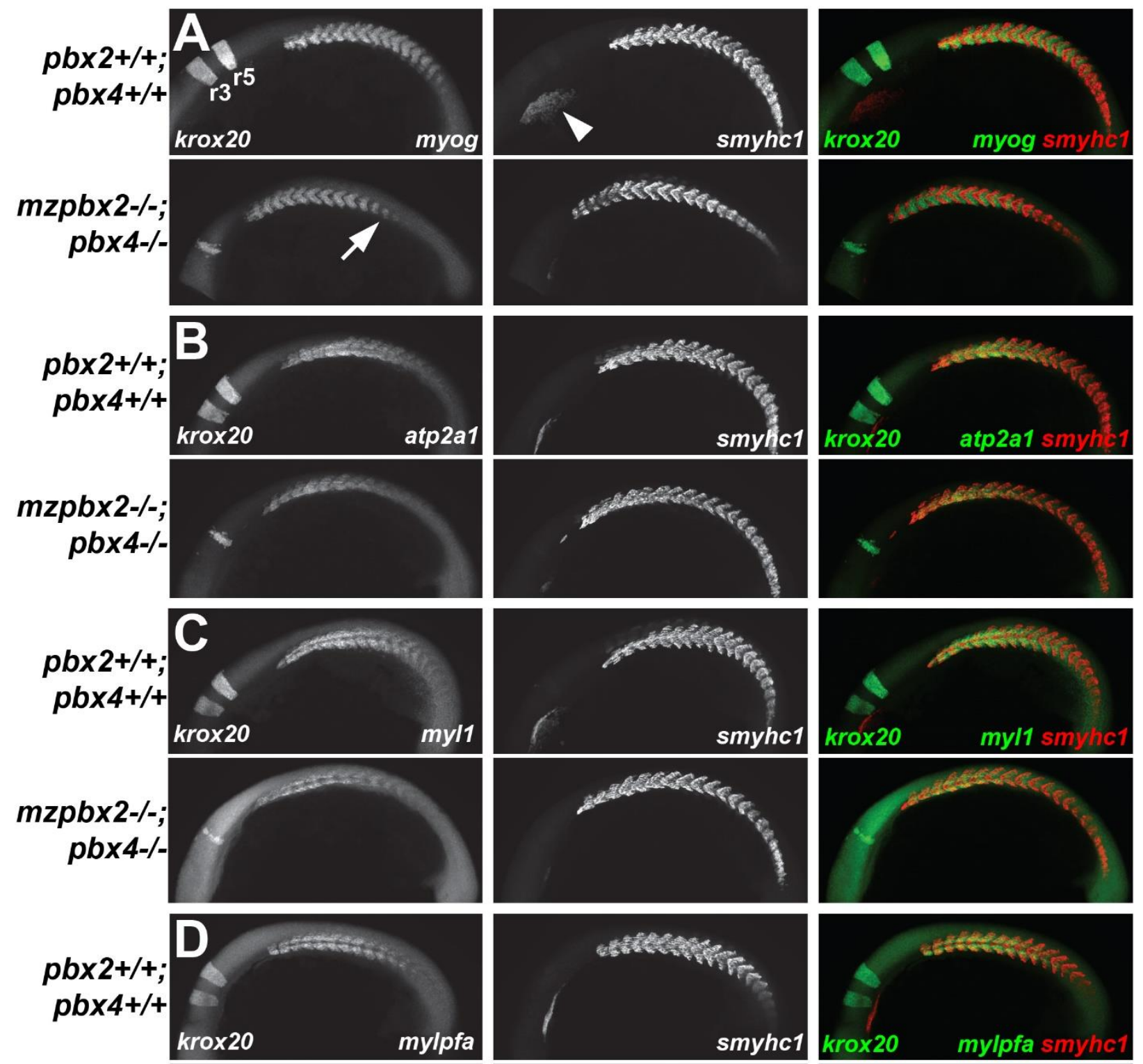

mzpbx2-/-; pbx4-/-
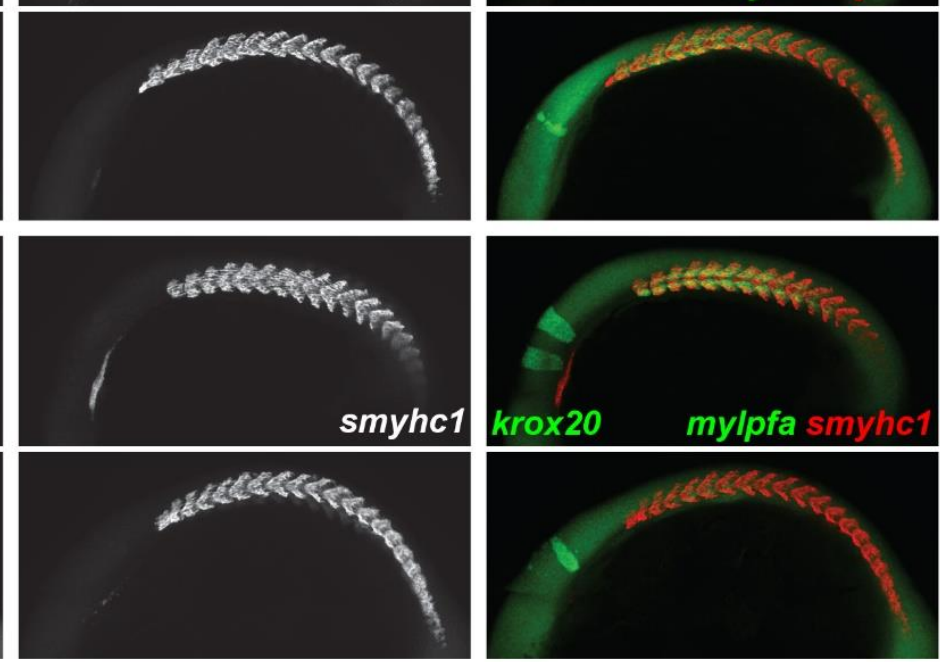
Figure 2
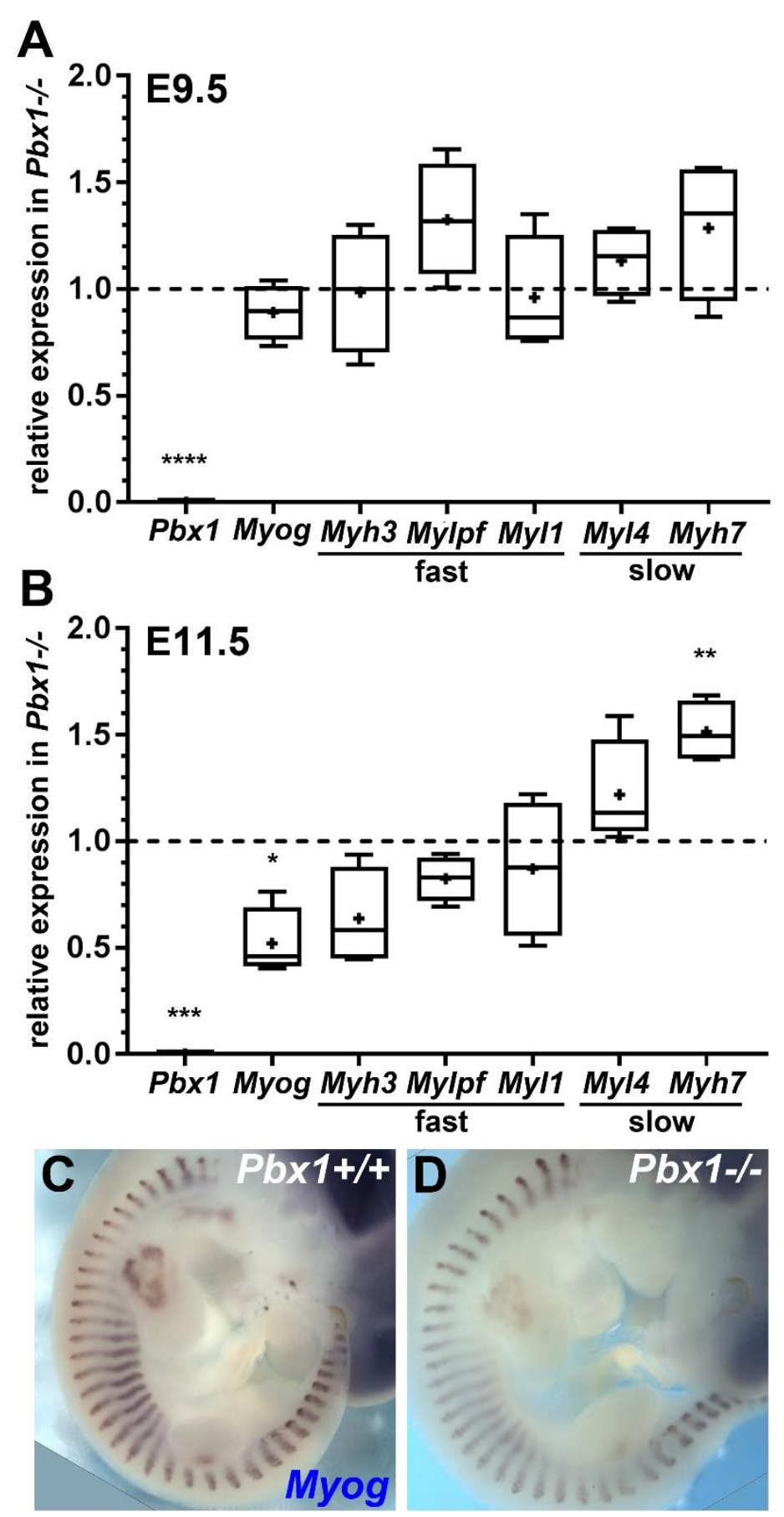
bioRxiv preprint doi: https://doi.org/10.1101/2020.02.21.960484; this version posted February 22, 2020. The copyright holder for this preprint (which was not certified by peer review) is the author/funder. All rights reserved. No reuse allowed without permission.

\section{Figure 3}
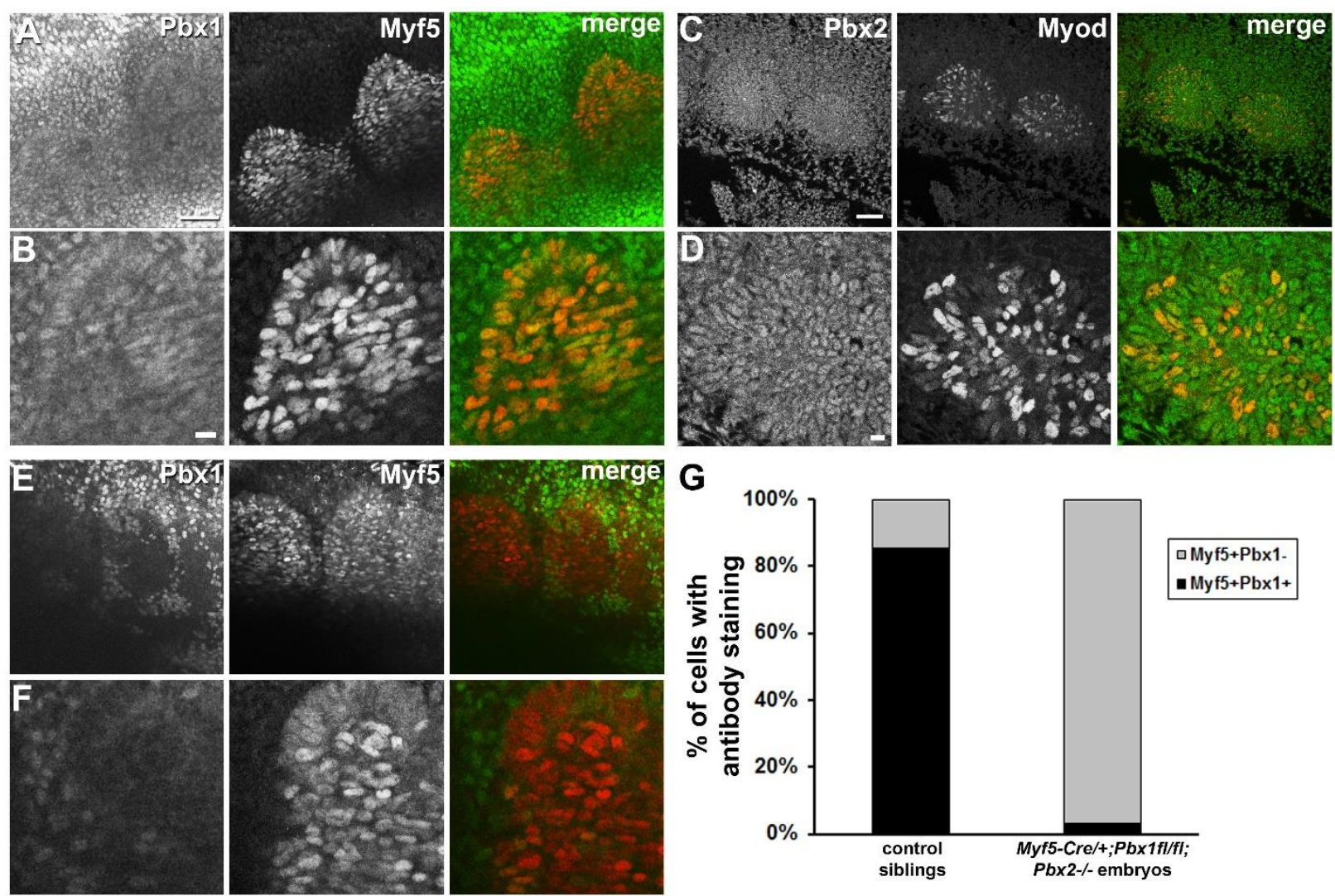
bioRxiv preprint doi: https://doi.org/10.1101/2020.02.21.960484; this version posted February 22, 2020. The copyright holder for this preprint (which was not certified by peer review) is the author/funder. All rights reserved. No reuse allowed without permission.

Figure 4
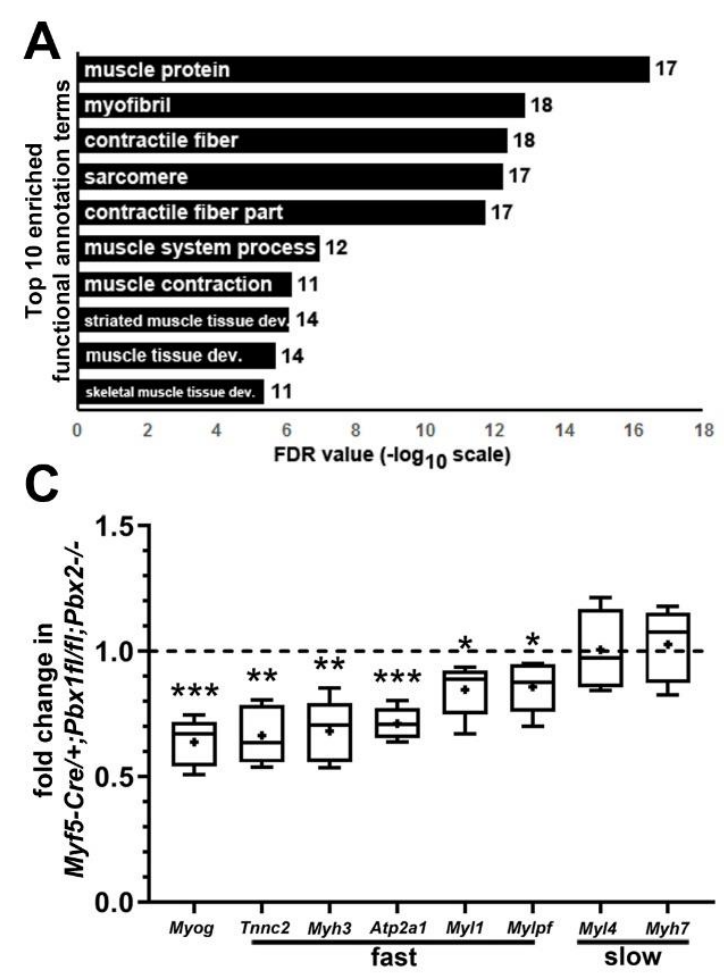

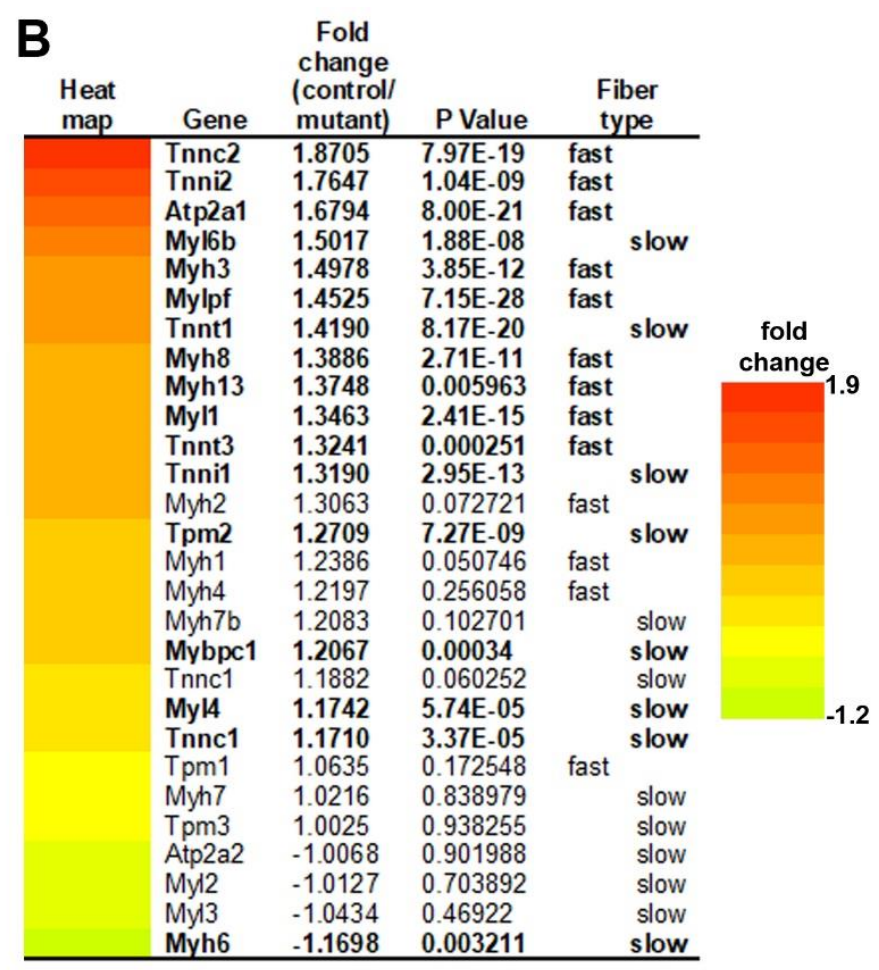


Figure 5

\section{E11.5}
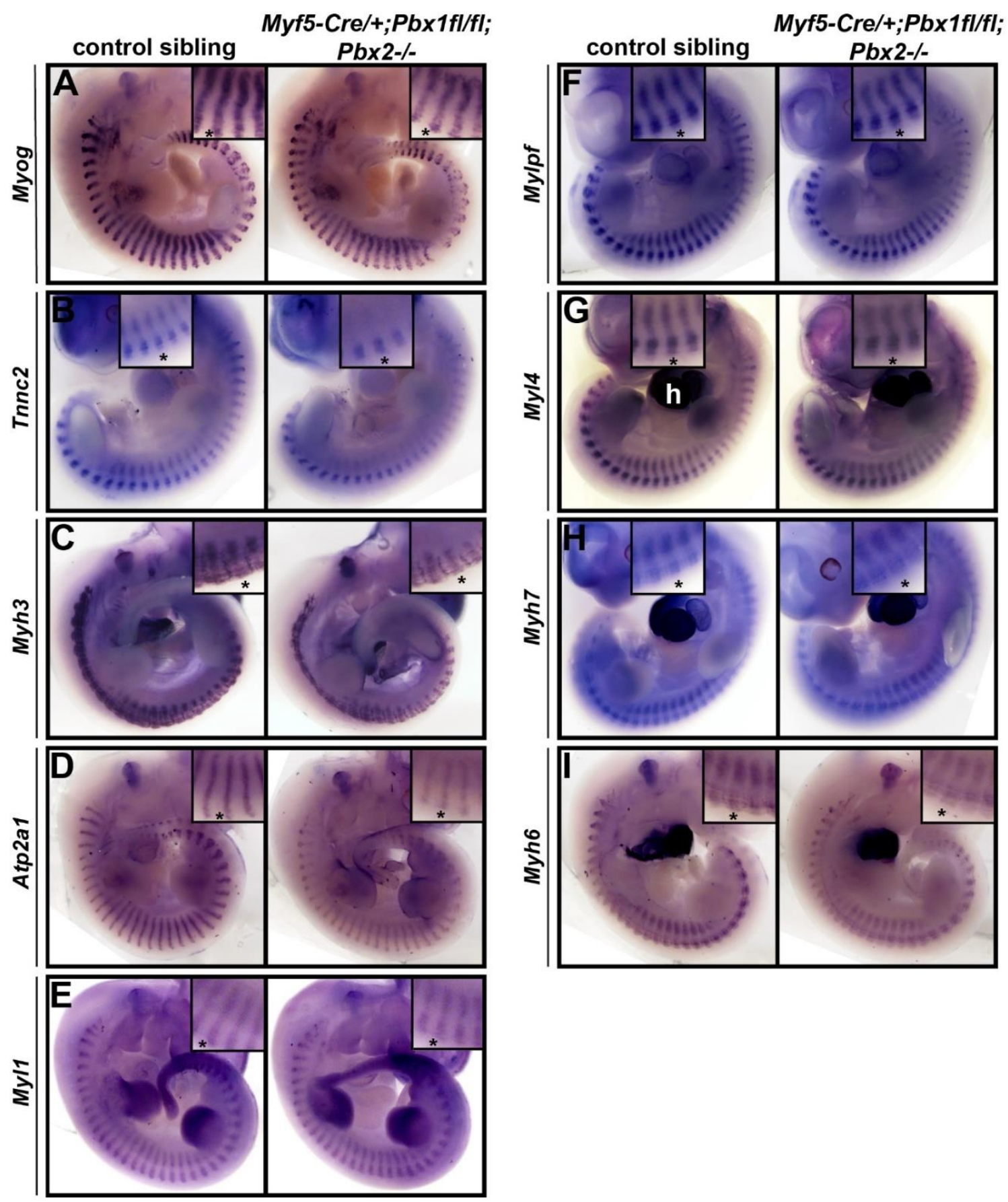


\title{
Supplemental Figure 1
}

\author{
A pbx2 WT AACATGCTGCTGGCT GAAGGTGTAGCCGGGCCAGAGAAAGGGGGCGGAGCCGCAGCGGCAG \\ pbx2 scm10 AACATGCTGCTGGCT GAAGGT GTAGCCGGGCCA GAaGAAAGGGGGCGGAGCCGCAGCGGCA
}
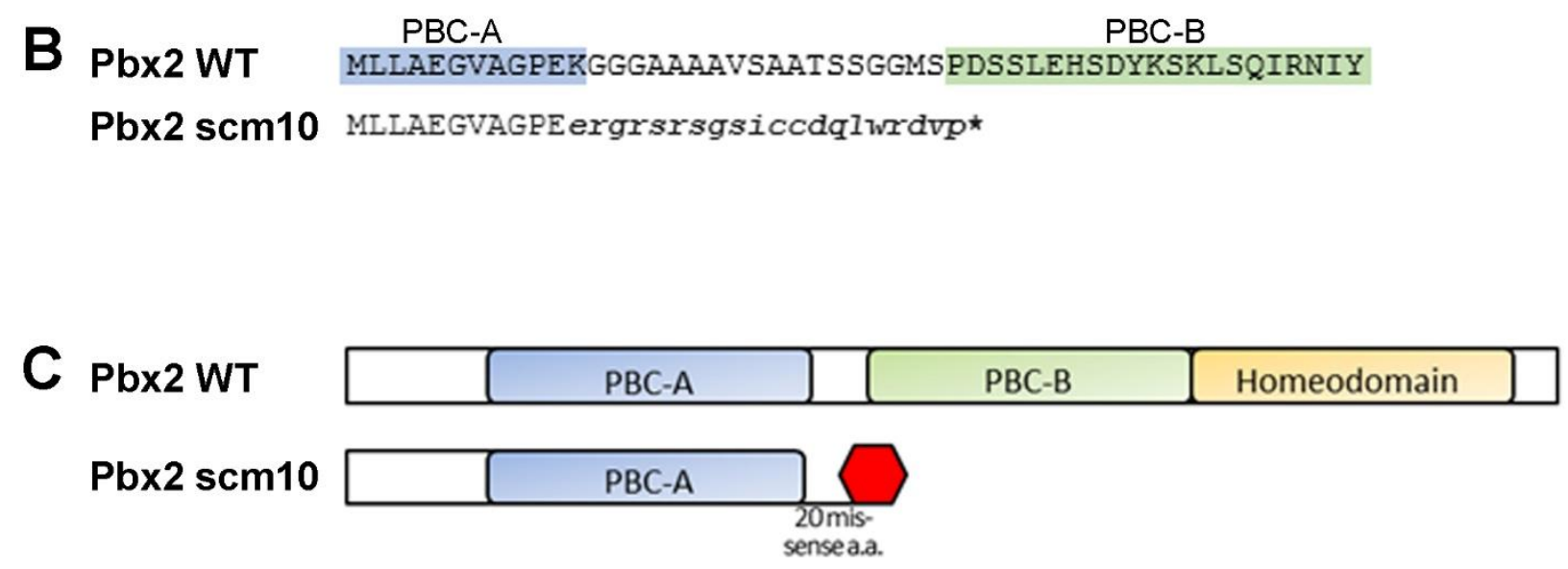

Supplemental Fig. 1. Generation of a zebrafish $p b x 2$ mutant allele with CRISPR/Cas9. (A) Top line: a portion of the wild-type (WT) genomic sequence of $p b x 2$ from the middle of Exon 3 showing the target sequence for the single-guide RNA (bold, underline) and the PAM sequence (green, bold). Bottom line: the sequence of the $p b x 2^{\text {scmlo }}$ allele with a single " $\mathrm{A}$ " nucleotide (highlighted) inserted 3bp upstream of the PAM. (B) Alignment of the conceptual translation of the $p b x 2^{s c m 10}$ allele compared to wild-type Pbx2. Mis-sense amino acids are in lower case and the premature STOP codon is noted with an asterisk. The PBC-A and -B domains are conserved among the Pbx family. (C) Cartoon showing the domain structure of Pbx2. The scm10 mutation is predicted to produce a truncated product lacking the conserved PBC-B and Homeobox domains and thus is likely to be a loss of function allele. 


\section{Supplemental Table 1}

\begin{tabular}{|c|c|c|c|}
\hline Ensembl Gene ID & Gene Symbol & log2FoldChange & PValue \\
\hline ENSMUSG00000059634 & Gm11942 & 1.997625366 & 0.00281949 \\
\hline ENSMUSG00000016327 & Atp1b4 & 1.251227808 & 1.02E-06 \\
\hline ENSMUSG00000073489 & Ifi204 & 1.124550446 & 0.00015198 \\
\hline ENSMUSG00000073643 & Wdfy1 & 1.123038354 & 4.92E-06 \\
\hline ENSMUSG00000074634 & Gm7120 & 1.119924629 & 0.00116979 \\
\hline ENSMUSG00000021758 & $\mathrm{Dd} \times 4$ & 1.102087178 & 0.00053919 \\
\hline ENSMUSG00000006542 & Prkag3 & 0.973063584 & 0.00075995 \\
\hline ENSMUSG00000019933 & 2310015B20Rik & 0.926526652 & 0.00014017 \\
\hline ENSMUSG00000017300 & Tnnc2 & 0.903462414 & 7.97E-19 \\
\hline ENSMUSG00000024578 & $\| 117 b$ & 0.900941787 & 9.94E-09 \\
\hline ENSMUSG00000085494 & 3110045C21Rik & 0.888868424 & 0.00015497 \\
\hline ENSMUSG00000025488 & Cox8b & 0.881086172 & 0.00501959 \\
\hline ENSMUSG00000025610 & ORF63 & 0.87635424 & 0.00059409 \\
\hline ENSMUSG00000087692 & Gm11940 & 0.875068601 & 0.00995758 \\
\hline ENSMUSG00000033044 & Dhrs7c & 0.83950002 & 0.00038984 \\
\hline ENSMUSG00000020429 & Igfbp1 & 0.83784793 & $1.94 \mathrm{E}-06$ \\
\hline ENSMUSG00000031097 & Tnni2 & 0.819467555 & 1.04E-09 \\
\hline ENSMUSG00000042828 & Trim72 & 0.817775515 & 2.70E-09 \\
\hline ENSMUSG00000074635 & 3110070M22Rik & 0.766527767 & 0.03872035 \\
\hline ENSMUSG00000062345 & Serpinb2 & 0.760078317 & 0.01888044 \\
\hline ENSMUSG00000057280 & Musk & 0.756434002 & 1.17E-06 \\
\hline ENSMUSG00000086866 & 4930512H18Rik & 0.753218651 & 0.00156951 \\
\hline ENSMUSG00000030730 & Atp2a1 & 0.748005332 & $8.00 E-21$ \\
\hline ENSMUSG00000085614 & 1700123M08Rik & 0.719703582 & 0.02952474 \\
\hline ENSMUSG00000020722 & Cacng1 & 0.701568796 & 0.00014105 \\
\hline ENSMUSG00000070645 & Ren1 & 0.695455825 & 0.00644048 \\
\hline ENSMUSG00000042895 & Abra & 0.693791851 & 0.02726867 \\
\hline ENSMUSG00000001508 & Sgca & 0.687273872 & 0.00187296 \\
\hline ENSMUSG00000062077 & Trim54 & 0.68587758 & 0.00055611 \\
\hline ENSMUSG00000033313 & Fbxl8 & 0.685161621 & 0.00346938 \\
\hline ENSMUSG00000042045 & SIn & 0.672438007 & $3.16 \mathrm{E}-07$ \\
\hline ENSMUSG00000026459 & Myog & 0.671548455 & $3.52 E-31$ \\
\hline ENSMUSG00000042451 & Mybph & 0.663330345 & 3.13E-21 \\
\hline ENSMUSG00000079363 & Gbp4 & 0.658339619 & 0.00629997 \\
\hline ENSMUSG00000025701 & Alox5 & 0.657609781 & 0.01013828 \\
\hline ENSMUSG00000090045 & Tmem229a & 0.655849126 & 0.02848229 \\
\hline ENSMUSG00000026574 & Dpt & 0.653574052 & 0.00189961 \\
\hline ENSMUSG00000040533 & Matn1 & 0.640553476 & 0.00617057 \\
\hline ENSMUSG00000028584 & Lrrc38 & 0.639594229 & 0.04050359 \\
\hline ENSMUSG00000022479 & Vdr & 0.637646441 & 0.00893736 \\
\hline
\end{tabular}




\begin{tabular}{|c|c|c|c|}
\hline ENSMUSG00000026725 & Tnn & 0.634312573 & 1.33E-06 \\
\hline ENSMUSG00000031461 & Myom2 & 0.628934127 & 8.06E-09 \\
\hline ENSMUSG00000034555 & Tex16 & 0.628500694 & 0.01715763 \\
\hline ENSMUSG00000048442 & 2210020M01Rik & 0.625970394 & 0.00461295 \\
\hline ENSMUSG00000042677 & Zc3h12a & 0.623725016 & 0.01930386 \\
\hline ENSMUSG00000025081 & Tdrd1 & 0.618142031 & $9.79 \mathrm{E}-05$ \\
\hline ENSMUSG00000070072 & Mir433 & 0.616452103 & 0.04237151 \\
\hline ENSMUSG00000044938 & KIhl31 & 0.616401756 & $1.16 \mathrm{E}-05$ \\
\hline ENSMUSG00000004038 & Gstm3 & 0.61330437 & 0.0094936 \\
\hline ENSMUSG00000002104 & Rapsn & 0.609688932 & $4.59 \mathrm{E}-07$ \\
\hline ENSMUSG00000071540 & 3425401B19Rik & 0.604462204 & 0.00091145 \\
\hline ENSMUSG00000055489 & Ano5 & 0.597258767 & 0.00092375 \\
\hline ENSMUSG00000038086 & Hspb2 & 0.589741021 & $2.88 \mathrm{E}-05$ \\
\hline ENSMUSG00000039824 & Myl6b & 0.586596978 & $1.88 \mathrm{E}-08$ \\
\hline ENSMUSG00000020908 & Myh3 & 0.582859624 & $3.85 \mathrm{E}-12$ \\
\hline ENSMUSG00000002007 & Srpk3 & 0.582406793 & 4.87E-05 \\
\hline ENSMUSG00000021211 & Akr1c12 & 0.580411755 & 0.02404366 \\
\hline ENSMUSG00000032860 & P2ry2 & 0.572441945 & 0.01732682 \\
\hline ENSMUSG00000021702 & Thbs4 & 0.566751503 & $8.09 E-15$ \\
\hline ENSMUSG00000005681 & Apoa2 & 0.565319962 & $6.79 \mathrm{E}-10$ \\
\hline ENSMUSG00000021680 & Crhbp & 0.565076794 & 0.01781884 \\
\hline ENSMUSG00000031074 & Fgf3 & 0.561368745 & 0.03998839 \\
\hline ENSMUSG00000058914 & C1qtnf3 & 0.560414393 & $6.26 \mathrm{E}-08$ \\
\hline ENSMUSG00000001260 & Gabrg1 & 0.560085266 & 0.01823623 \\
\hline ENSMUSG00000055994 & Nod2 & 0.558336216 & 0.03278935 \\
\hline ENSMUSG00000020583 & Matn3 & 0.555032035 & 0.00975114 \\
\hline ENSMUSG00000000435 & Myf5 & 0.554993541 & 0.00097489 \\
\hline ENSMUSG00000026100 & Mstn & 0.554436286 & $3.49 E-05$ \\
\hline ENSMUSG00000044086 & Lmod3 & 0.553440981 & 3.07E-05 \\
\hline ENSMUSG00000031932 & Gpr83 & 0.550355575 & 0.01823693 \\
\hline ENSMUSG00000053063 & Clec12a & 0.550353594 & 0.02898404 \\
\hline ENSMUSG00000024471 & Myot & 0.54995695 & 3.09E-05 \\
\hline ENSMUSG00000009214 & Tmem8c & 0.544022249 & $9.25 \mathrm{E}-08$ \\
\hline ENSMUSG00000049565 & Aknad1 & 0.54361441 & 0.02271281 \\
\hline ENSMUSG00000058715 & Fcer1g & 0.542167393 & 0.00021779 \\
\hline ENSMUSG00000026971 & Itgb6 & 0.53923265 & 0.00141368 \\
\hline ENSMUSG00000030672 & Mylpf & 0.538601704 & $7.15 E-28$ \\
\hline ENSMUSG00000028414 & Fktn & 0.533896702 & 2.46E-08 \\
\hline ENSMUSG00000074882 & Сyp2c68 & 0.529203976 & 0.00604621 \\
\hline ENSMUSG00000079173 & Zan & 0.526269092 & 0.03147969 \\
\hline ENSMUSG00000038354 & Ankrd35 & 0.521552035 & 0.02320364 \\
\hline ENSMUSG00000022215 & Fitm1 & 0.52050739 & 0.00029212 \\
\hline ENSMUSG00000045589 & 6430704M03Rik & 0.511679318 & $2.21 \mathrm{E}-05$ \\
\hline ENSMUSG00000039376 & Synpo2l & 0.511592176 & $2.50 \mathrm{E}-12$ \\
\hline ENSMUSG00000026950 & Neb & 0.509892859 & 8.69E-08 \\
\hline ENSMUSG00000045404 & Kcnk13 & 0.508874051 & 0.00519331 \\
\hline
\end{tabular}




\begin{tabular}{|c|c|c|c|}
\hline ENSMUSG00000031972 & Acta1 & 0.50797014 & $4.86 \mathrm{E}-16$ \\
\hline ENSMUSG00000064179 & Tnnt1 & 0.504897667 & 8.17E-20 \\
\hline ENSMUSG00000062515 & Fabp4 & 0.502155826 & 0.01847878 \\
\hline ENSMUSG00000073490 & Al607873 & 0.498061198 & 0.0179164 \\
\hline ENSMUSG00000041828 & Abca8a & 0.497036606 & 0.003378 \\
\hline ENSMUSG00000045259 & Klhdc9 & 0.496090908 & 0.00044735 \\
\hline ENSMUSG00000028758 & Kif17 & 0.495159619 & 0.04926557 \\
\hline ENSMUSG00000059775 & Rps26-ps1 & 0.490654785 & 0.00238278 \\
\hline ENSMUSG00000007682 & Dio2 & 0.488216039 & 0.01209769 \\
\hline ENSMUSG00000028396 & 2310002L09Rik & 0.484231204 & 0.01232068 \\
\hline ENSMUSG00000031962 & Cdh15 & 0.477951759 & $7.25 \mathrm{E}-08$ \\
\hline ENSMUSG00000035606 & Ky & 0.475709302 & 0.00504494 \\
\hline ENSMUSG00000042429 & Adora1 & 0.474981096 & $5.98 \mathrm{E}-07$ \\
\hline ENSMUSG00000055775 & Myh8 & 0.473679617 & $2.71 \mathrm{E}-11$ \\
\hline ENSMUSG00000084897 & Gm14226 & 0.469395994 & 0.02938826 \\
\hline ENSMUSG00000036855 & Gjd4 & 0.469300148 & 0.01647953 \\
\hline ENSMUSG00000074001 & Kbtbd5 & 0.467147461 & 0.00015936 \\
\hline ENSMUSG00000078521 & 2610002D18Rik & 0.462381336 & 0.04991346 \\
\hline ENSMUSG00000027861 & Casq2 & 0.462242954 & $1.43 E-08$ \\
\hline ENSMUSG00000060180 & Myh13 & 0.459267373 & 0.00596266 \\
\hline ENSMUSG00000036214 & Znrd1as & 0.452895392 & 0.01072842 \\
\hline ENSMUSG00000020787 & P2rx1 & 0.452229276 & 0.01509239 \\
\hline ENSMUSG00000086327 & 1700003F17Rik & 0.451494344 & 0.01304189 \\
\hline ENSMUSG00000025450 & Gm9752 & 0.448148532 & 0.02543087 \\
\hline ENSMUSG00000020067 & Mypn & 0.447078889 & 0.00166056 \\
\hline ENSMUSG00000047631 & Apof & 0.444096313 & 0.02441692 \\
\hline ENSMUSG00000075307 & Kbtbd10 & 0.443384263 & $5.80 \mathrm{E}-07$ \\
\hline ENSMUSG00000041556 & Fbxo2 & 0.442022852 & 0.03041434 \\
\hline ENSMUSG00000037086 & 1110059M19Rik & 0.439579622 & 0.00588576 \\
\hline ENSMUSG00000085236 & 2610206C17Rik & 0.435065022 & 0.02246406 \\
\hline ENSMUSG00000023993 & Treml1 & 0.433037879 & 0.01750325 \\
\hline ENSMUSG00000062822 & 4833420G17Rik & 0.432153462 & 0.00041592 \\
\hline ENSMUSG00000038403 & $\mathrm{Hfe} 2$ & 0.430554005 & 0.00018901 \\
\hline ENSMUSG00000061816 & Myl1 & 0.429046152 & $2.41 \mathrm{E}-15$ \\
\hline ENSMUSG00000040287 & Stac3 & 0.42305648 & 0.00195412 \\
\hline ENSMUSG00000019936 & Epyc & 0.422547599 & 0.00393017 \\
\hline ENSMUSG00000018920 & Cxcl16 & 0.421573156 & 0.0159116 \\
\hline ENSMUSG00000043687 & 1190005I06Rik & 0.421154892 & 0.02896011 \\
\hline ENSMUSG00000006403 & Adamts4 & 0.417012512 & 0.00010599 \\
\hline ENSMUSG00000043789 & Vwce & 0.416472145 & 0.01681858 \\
\hline ENSMUSG00000026390 & Marco & 0.416332664 & 0.01417638 \\
\hline ENSMUSG00000000290 & Itgb2 & 0.415374413 & 0.01923706 \\
\hline ENSMUSG00000052374 & Actn2 & 0.415039379 & 7.07E-05 \\
\hline ENSMUSG00000031312 & Itgb1bp2 & 0.410967671 & 0.0004314 \\
\hline ENSMUSG00000057606 & Colq & 0.407707638 & 0.03184891 \\
\hline ENSMUSG00000042485 & Mustn1 & 0.40547905 & 0.00715716 \\
\hline
\end{tabular}




\begin{tabular}{|c|c|c|c|}
\hline ENSMUSG00000047011 & Mtap4 & 0.405248997 & 0.0176333 \\
\hline ENSMUSG00000061723 & Tnnt3 & 0.405037407 & 0.00025145 \\
\hline ENSMUSG00000031618 & $\mathrm{Nr} 3 \mathrm{c} 2$ & 0.401968702 & 0.01138455 \\
\hline ENSMUSG00000021200 & Asb2 & 0.401899298 & 0.00062097 \\
\hline ENSMUSG00000026418 & Tnni1 & 0.399544026 & $2.95 \mathrm{E}-13$ \\
\hline ENSMUSG00000031765 & Mt1 & 0.396005941 & $6.60 \mathrm{E}-12$ \\
\hline ENSMUSG00000028417 & Tal2 & 0.393486658 & 0.02103196 \\
\hline ENSMUSG00000051747 & Ttn & 0.393208884 & 0.00015848 \\
\hline ENSMUSG00000024887 & Asah2 & 0.391290823 & 0.00251144 \\
\hline ENSMUSG00000027188 & Pamr1 & 0.390065372 & 3.59E-05 \\
\hline ENSMUSG00000025229 & Pitx3 & 0.38984228 & 0.00477099 \\
\hline ENSMUSG00000060791 & Gmfg & 0.389490721 & 0.02270391 \\
\hline ENSMUSG00000020216 & Jsrp1 & 0.38913719 & 0.03574297 \\
\hline ENSMUSG00000028470 & Hint2 & 0.388875622 & 0.00196966 \\
\hline ENSMUSG00000079110 & Capn3 & 0.388867779 & 0.03968014 \\
\hline ENSMUSG00000040694 & Apobec2 & 0.38794292 & 0.00061565 \\
\hline ENSMUSG00000028116 & Myoz2 & 0.387903641 & 0.00293157 \\
\hline ENSMUSG00000086566 & 2810429I04Rik & 0.387281461 & 0.01446724 \\
\hline ENSMUSG00000029372 & Ppbp & 0.386538655 & 0.00688941 \\
\hline ENSMUSG00000001334 & Fndc5 & 0.385176976 & 7.31E-08 \\
\hline ENSMUSG00000086741 & Gm15816 & 0.384866487 & 0.00192303 \\
\hline ENSMUSG00000038763 & Alpk3 & 0.383775593 & 0.00091421 \\
\hline ENSMUSG00000031762 & Mt2 & 0.380790014 & 1.05E-08 \\
\hline ENSMUSG00000010609 & Psen2 & 0.37909022 & 0.00164963 \\
\hline ENSMUSG00000062329 & Cytl1 & 0.375253939 & 0.00945664 \\
\hline ENSMUSG00000029925 & Tbxas1 & 0.37494231 & 0.04030196 \\
\hline ENSMUSG00000021388 & Aspn & 0.374378291 & 0.00264657 \\
\hline ENSMUSG00000041189 & Chrnb1 & 0.373828981 & 0.00568736 \\
\hline ENSMUSG00000030785 & Cox6a2 & 0.369946973 & 0.00490543 \\
\hline ENSMUSG00000026678 & Rgs5 & 0.368894323 & 0.0001231 \\
\hline ENSMUSG00000064361 & $\mathrm{mt}-\mathrm{Tr}$ & 0.367063972 & 0.01987099 \\
\hline ENSMUSG00000030607 & Acan & 0.365270209 & 0.00602465 \\
\hline ENSMUSG00000036278 & Macrod1 & 0.362187382 & 0.00858772 \\
\hline ENSMUSG00000030996 & Art1 & 0.357918724 & 0.04952297 \\
\hline ENSMUSG00000079471 & Gm7325 & 0.356200875 & 0.00371319 \\
\hline ENSMUSG00000053963 & 6330403A02Rik & 0.355211687 & 0.00294897 \\
\hline ENSMUSG00000032925 & Itgbl1 & 0.348910538 & 0.03793757 \\
\hline ENSMUSG00000028464 & Tpm2 & 0.345856658 & 7.27E-09 \\
\hline ENSMUSG00000027487 & Cdk5rap1 & 0.345253026 & 0.0072262 \\
\hline ENSMUSG00000027107 & Chrna1 & 0.344822171 & 0.00128325 \\
\hline ENSMUSG00000037318 & Traf3ip3 & 0.342951693 & 0.00952413 \\
\hline ENSMUSG00000079428 & Tceal7 & 0.34187202 & 0.00031241 \\
\hline ENSMUSG00000052305 & Hbb-b1 & 0.340499141 & 2.47E-06 \\
\hline ENSMUSG00000023067 & Cdkn1a & 0.338367609 & 2.87E-06 \\
\hline ENSMUSG00000024330 & Col11a2 & 0.337093452 & 0.00588635 \\
\hline ENSMUSG00000036249 & Rbm43 & 0.333843039 & 0.00682483 \\
\hline
\end{tabular}




\begin{tabular}{|c|c|c|c|}
\hline ENSMUSG00000087107 & Al662270 & 0.333264522 & 0.03271734 \\
\hline ENSMUSG00000086596 & Susd5 & 0.330333555 & 0.02086009 \\
\hline ENSMUSG00000044951 & Mylk4 & 0.330267863 & 0.00592378 \\
\hline ENSMUSG00000027199 & Gatm & 0.32912307 & 0.00033889 \\
\hline ENSMUSG00000035296 & Sgcg & 0.327349369 & 0.03261574 \\
\hline ENSMUSG00000068122 & Agtr2 & 0.320310535 & $1.00 \mathrm{E}-05$ \\
\hline ENSMUSG00000025076 & Casp7 & 0.31925688 & 0.00219929 \\
\hline ENSMUSG00000055027 & Smyd1 & 0.316585047 & 7.11E-06 \\
\hline ENSMUSG00000004730 & Emr1 & 0.315186362 & 0.00499263 \\
\hline ENSMUSG00000068614 & Actc1 & 0.313852677 & $9.43 \mathrm{E}-07$ \\
\hline ENSMUSG00000009246 & Trpm5 & 0.313253164 & 0.0009709 \\
\hline ENSMUSG00000026407 & Cacna1s & 0.311114329 & 0.01526524 \\
\hline ENSMUSG00000073940 & $\mathrm{Hbb}-\mathrm{b} 2$ & 0.309187296 & 4.58E-05 \\
\hline ENSMUSG00000067235 & H2-Q10 & 0.309078837 & 0.02397856 \\
\hline ENSMUSG00000057457 & Phex & 0.308873813 & 0.01362792 \\
\hline ENSMUSG00000047910 & Pcdhb16 & 0.307913156 & 0.03913632 \\
\hline ENSMUSG00000024427 & Spry4 & 0.307038996 & 0.00012221 \\
\hline ENSMUSG00000043518 & Rai2 & 0.304910395 & 0.00774812 \\
\hline ENSMUSG00000021798 & Ldb3 & 0.304832892 & 0.00714162 \\
\hline ENSMUSG00000074818 & Pdzd7 & 0.304509786 & 0.01792369 \\
\hline ENSMUSG00000060913 & Trim55 & 0.30356309 & 0.01127983 \\
\hline ENSMUSG00000023885 & Thbs2 & 0.301062714 & $1.91 \mathrm{E}-07$ \\
\hline ENSMUSG00000090066 & 1110002E22Rik & 0.299868154 & 0.01615601 \\
\hline ENSMUSG00000048612 & Myof & 0.297460533 & $1.38 \mathrm{E}-06$ \\
\hline ENSMUSG00000011148 & Adss 11 & 0.295776045 & 0.02495573 \\
\hline ENSMUSG00000029084 & Cd38 & 0.294192572 & 0.01011198 \\
\hline ENSMUSG00000054484 & Tmem62 & 0.293070552 & 0.04439612 \\
\hline ENSMUSG00000032503 & Arpp21 & 0.290096675 & 0.00241346 \\
\hline ENSMUSG00000025068 & Gsto1 & 0.286751633 & 0.00090928 \\
\hline ENSMUSG00000032735 & Ablim3 & 0.285535424 & 2.73E-06 \\
\hline ENSMUSG00000089902 & Gm13625 & 0.284828783 & 0.04901378 \\
\hline ENSMUSG00000038239 & $\mathrm{Hrc}$ & 0.284401846 & 0.02072195 \\
\hline ENSMUSG00000069919 & Hba-a1 & 0.284350875 & 0.00116311 \\
\hline ENSMUSG00000020475 & Pgam2 & 0.283618949 & 0.00943925 \\
\hline ENSMUSG00000069917 & Hba-a2 & 0.282161757 & 0.00129652 \\
\hline ENSMUSG00000025017 & Pik3ap1 & 0.282020849 & 0.02903027 \\
\hline ENSMUSG00000002992 & Apoc2 & 0.279395317 & 0.01275751 \\
\hline ENSMUSG00000002944 & Cd36 & 0.279227521 & 0.02499893 \\
\hline ENSMUSG00000060600 & Eno3 & 0.27803025 & 0.00058382 \\
\hline ENSMUSG00000020150 & Gamt & 0.277184632 & 0.00685728 \\
\hline ENSMUSG00000038132 & Rbm24 & 0.276930268 & 0.00133199 \\
\hline ENSMUSG00000001493 & Meox1 & 0.275090349 & 0.00313707 \\
\hline ENSMUSG00000053684 & BC048403 & 0.274289185 & 0.03833233 \\
\hline ENSMUSG00000022025 & Lect1 & 0.27417929 & 0.01631824 \\
\hline ENSMUSG00000052160 & Pld4 & 0.271590944 & 0.03456755 \\
\hline ENSMUSG00000054150 & 4831426l19Rik & 0.271509199 & 0.04166042 \\
\hline
\end{tabular}


bioRxiv preprint doi: https://doi.org/10.1101/2020.02.21.960484; this version posted February 22, 2020. The copyright holder for this preprint (which was not certified by peer review) is the author/funder. All rights reserved. No reuse allowed without permission.

\begin{tabular}{llll} 
ENSMUSG00000020061 & Mybpc1 & 0.271137725 & 0.00033988 \\
ENSMUSG00000064349 & mt-Tc & 0.269482522 & 0.01920889 \\
ENSMUSG00000049538 & Adamts16 & 0.267382379 & 0.02498982 \\
ENSMUSG00000043903 & Gm22 & 0.264526387 & 0.02548835 \\
ENSMUSG00000028736 & Pax7 & 0.263736512 & 0.01444815 \\
\hline
\end{tabular}




\section{Supplemental Table 2}

Table S2 Genes upregulated in $\mathrm{Myf5}^{\mathrm{Cre} /+} ; \mathrm{Pbx} 1^{\mathrm{fl/fl} ;} \mathrm{Pbx2}^{-/-} \mathrm{E} 11.5$ embryos $(\mathrm{FC}<-1.2, \mathrm{P}<0.05)$

\begin{tabular}{|c|c|c|c|}
\hline Ensembl Gene ID & Gene Symbol & log2FoldChange & PValue \\
\hline ENSMUSG00000032080 & Apoa4 & -6.306315557 & $3.17 E-14$ \\
\hline ENSMUSG00000074373 & Gm10680 & -5.515828015 & $1.72 \mathrm{E}-11$ \\
\hline ENSMUSG00000078503 & Gm13225 & -5.47922862 & $7.48 \mathrm{E}-11$ \\
\hline ENSMUSG00000040808 & $\mathrm{S} 100 \mathrm{~g}$ & -3.325509795 & $1.54 \mathrm{E}-05$ \\
\hline ENSMUSG00000025172 & Ankrd2 & -2.928952639 & $5.43 E-13$ \\
\hline ENSMUSG00000062518 & Zfp534 & -2.538250615 & 0.00029127 \\
\hline ENSMUSG00000031410 & Nxf7 & -2.326189513 & 2.23E-06 \\
\hline ENSMUSG00000064193 & Gm13699 & -1.862530812 & $2.50 \mathrm{E}-05$ \\
\hline ENSMUSG00000085715 & Tsix & -1.64619603 & 0.003453398 \\
\hline ENSMUSG00000026726 & Cubn & -1.588274999 & 0.000301274 \\
\hline ENSMUSG00000086503 & Xist & -1.42707307 & 0.00966999 \\
\hline ENSMUSG00000042357 & Gjb5 & -1.389972315 & 0.000216672 \\
\hline ENSMUSG00000031098 & Syt8 & -1.22251762 & 0.004651279 \\
\hline ENSMUSG00000025400 & Tac2 & -1.208829904 & 0.009045378 \\
\hline ENSMUSG00000026535 & Ifi202b & -1.190125984 & 0.00012606 \\
\hline ENSMUSG00000021278 & Amn & -1.118509657 & 0.004797454 \\
\hline ENSMUSG00000034427 & Myo15b & -1.117488525 & 0.002139268 \\
\hline ENSMUSG00000063011 & Msln & -1.024260596 & 0.010304563 \\
\hline ENSMUSG00000048528 & Nkx1-2 & -1.018518435 & 0.000299412 \\
\hline ENSMUSG00000048087 & Gm4737 & -0.98277365 & 0.012815925 \\
\hline ENSMUSG00000048538 & Gm9826 & -0.965443669 & 0.005130603 \\
\hline ENSMUSG00000049908 & Gja8 & -0.887394669 & 0.007521134 \\
\hline ENSMUSG00000074000 & Gm10606 & -0.823043723 & 0.007204508 \\
\hline ENSMUSG00000029195 & $\mathrm{Klb}$ & -0.803722238 & 0.001863233 \\
\hline ENSMUSG00000086219 & 2410137F16Rik & -0.797311711 & 0.003548871 \\
\hline ENSMUSG00000073125 & $\mathrm{XIr3b}$ & -0.780298726 & 0.000136367 \\
\hline ENSMUSG00000087130 & D230004N17Rik & -0.778579788 & 0.02928641 \\
\hline ENSMUSG00000070900 & Gm10306 & -0.76539987 & 0.002570925 \\
\hline ENSMUSG00000057836 & XIr3a & -0.759084446 & 0.000858649 \\
\hline ENSMUSG00000075589 & Gm11536 & -0.741431847 & 0.019912768 \\
\hline ENSMUSG00000086983 & Gm16972 & -0.720476111 & 0.002334959 \\
\hline ENSMUSG00000091344 & 1700037F24Rik & -0.716684418 & 0.017844048 \\
\hline ENSMUSG00000036279 & Zbtb20 & -0.709206915 & 0.005800701 \\
\hline ENSMUSG00000090300 & Gm17119 & -0.699302536 & 0.025911127 \\
\hline ENSMUSG00000087126 & 1700109K24Rik & -0.695473973 & 0.015247948 \\
\hline ENSMUSG00000020609 & Apob & -0.689822562 & 0.006034631 \\
\hline ENSMUSG00000087445 & Gm14286 & -0.682320993 & 0.006918723 \\
\hline ENSMUSG00000058147 & XIr3c & -0.663193337 & 0.007801348 \\
\hline ENSMUSG00000025905 & Oprk1 & -0.662227666 & 0.034090499 \\
\hline
\end{tabular}




\begin{tabular}{|c|c|c|c|}
\hline ENSMUSG00000064797 & snoZ40 & -0.654614859 & 0.036797891 \\
\hline ENSMUSG00000003354 & Ccdc65 & -0.645778509 & 0.019246502 \\
\hline ENSMUSG00000088524 & Snord2 & -0.630430525 & 0.041410335 \\
\hline ENSMUSG00000026175 & Vil1 & -0.612509025 & 0.024694391 \\
\hline ENSMUSG00000081382 & Rpl18-ps1 & -0.600675167 & 0.014347111 \\
\hline ENSMUSG00000026656 & Fcgr2b & -0.594487947 & 0.029671309 \\
\hline ENSMUSG00000013611 & Snx31 & -0.590706189 & 0.041840498 \\
\hline ENSMUSG00000078736 & Gm2023 & -0.586318407 & 0.015163515 \\
\hline ENSMUSG00000086903 & Gm16258 & -0.577412052 & 0.014299005 \\
\hline ENSMUSG00000052450 & 2810055G20Rik & -0.56102587 & 0.006218148 \\
\hline ENSMUSG00000043773 & 1700048O20Rik & -0.552712293 & 0.035969575 \\
\hline ENSMUSG00000085144 & A930029G22Rik & -0.550742938 & 0.027274843 \\
\hline ENSMUSG00000073374 & C030034I22Rik & -0.545615984 & 0.02575865 \\
\hline ENSMUSG00000058773 & Hist1h1b & -0.54060025 & 0.043517707 \\
\hline ENSMUSG00000071550 & Wdr52 & -0.538737931 & 0.038204208 \\
\hline ENSMUSG00000071005 & Ccl19 & -0.537158827 & 0.031752102 \\
\hline ENSMUSG00000034472 & Rasd2 & -0.526510352 & 0.035130209 \\
\hline ENSMUSG00000006143 & 2310043J07Rik & -0.524123792 & 0.028261176 \\
\hline ENSMUSG00000087490 & A330076H08Rik & -0.520190635 & 0.015905084 \\
\hline ENSMUSG00000073877 & Gm13306 & -0.517113532 & 0.001602514 \\
\hline ENSMUSG00000043488 & Gm9783 & -0.51683802 & 0.032243702 \\
\hline ENSMUSG00000037660 & Gdf7 & -0.513695846 & 0.017432151 \\
\hline ENSMUSG00000075296 & Aldh3b2 & -0.51265723 & 0.027387874 \\
\hline ENSMUSG00000082585 & Gm15387 & -0.512017147 & 0.026753475 \\
\hline ENSMUSG00000040148 & $\mathrm{Hm} \times 3$ & -0.510956447 & 0.048865905 \\
\hline ENSMUSG00000011008 & Mcoln2 & -0.508964718 & 0.033444389 \\
\hline ENSMUSG00000066202 & Ccl27b & -0.508429922 & 0.001916313 \\
\hline ENSMUSG00000086103 & Gm11832 & -0.505445927 & 0.048687777 \\
\hline ENSMUSG00000050328 & Hoxc12 & -0.504155076 & 0.004718282 \\
\hline ENSMUSG00000078734 & Gm2506 & -0.499562449 & 0.00232082 \\
\hline ENSMUSG00000061991 & Hist1h2af & -0.499256211 & 0.022297201 \\
\hline ENSMUSG00000091455 & Otogl & -0.498867697 & 0.036221036 \\
\hline ENSMUSG00000078747 & Gm13308 & -0.492689343 & 0.005162536 \\
\hline ENSMUSG00000034570 & Inpp5j & -0.482996943 & 0.037139714 \\
\hline ENSMUSG00000093629 & RP24-89J3.3.1 & -0.482861525 & 0.032686405 \\
\hline ENSMUSG00000086843 & E030013I19Rik & -0.482844259 & 0.002581848 \\
\hline ENSMUSG00000027070 & Lrp2 & -0.481726552 & 0.005171399 \\
\hline ENSMUSG00000064968 & Snord47 & -0.47430961 & 0.019388459 \\
\hline ENSMUSG00000048015 & Neurod4 & -0.453916921 & 0.00827315 \\
\hline ENSMUSG00000033200 & Tpsg1 & -0.452988883 & 0.037538228 \\
\hline ENSMUSG00000025176 & Hoga1 & -0.448387514 & 0.013597914 \\
\hline ENSMUSG00000072572 & Slc39a2 & -0.441203678 & 0.029232621 \\
\hline ENSMUSG00000090454 & Gm17341 & -0.439560692 & 0.04550548 \\
\hline ENSMUSG00000060470 & Gpr97 & -0.437686664 & 0.00936483 \\
\hline ENSMUSG00000039110 & Mycbpap & -0.436628091 & 0.038426709 \\
\hline
\end{tabular}




\begin{tabular}{|c|c|c|c|}
\hline ENSMUSG00000031450 & Grk1 & -0.431999342 & 0.049762882 \\
\hline ENSMUSG00000085730 & Gm13307 & -0.430442756 & 0.035219898 \\
\hline ENSMUSG00000073888 & Ccl27a & -0.429593487 & 8.06E-05 \\
\hline ENSMUSG00000073442 & Gm16386 & -0.429037138 & 0.03385672 \\
\hline ENSMUSG00000092827 & Mir3091 & -0.427362633 & 0.021540545 \\
\hline ENSMUSG00000071033 & Gm10308 & -0.418041797 & 0.036558743 \\
\hline ENSMUSG00000089417 & snoU90 & -0.413098433 & 0.001259728 \\
\hline ENSMUSG00000081669 & Npm3-ps1 & -0.412365229 & 0.00186434 \\
\hline ENSMUSG00000051977 & Prdm9 & -0.410887138 & 0.045070606 \\
\hline ENSMUSG00000034145 & Tmem63c & -0.406131619 & 0.032119561 \\
\hline ENSMUSG00000028392 & Bspry & -0.402511396 & 0.018211081 \\
\hline ENSMUSG00000036078 & Sigmar1 & -0.402257195 & 0.000247912 \\
\hline ENSMUSG00000038422 & Hdhd3 & -0.401825763 & 0.004742039 \\
\hline ENSMUSG00000090386 & 2810055G20Rik & -0.401158534 & 0.026295633 \\
\hline ENSMUSG00000066201 & Il11ra2 & -0.399345083 & 0.000443053 \\
\hline ENSMUSG00000073876 & Gm13305 & -0.398860782 & 0.000567427 \\
\hline ENSMUSG00000078735 & Gm2002 & -0.397553566 & 0.000735166 \\
\hline ENSMUSG00000084869 & Gm13063 & -0.391921682 & 0.010075281 \\
\hline ENSMUSG00000034258 & Mfsd7c & -0.385244072 & 0.020670293 \\
\hline ENSMUSG00000092901 & Mir5130 & -0.374405672 & 0.022756003 \\
\hline ENSMUSG00000093485 & RP23-268N22.1.1 & -0.372013329 & 0.03427877 \\
\hline ENSMUSG00000020159 & Gabrp & -0.367993171 & 0.012219199 \\
\hline ENSMUSG00000037649 & $\mathrm{H} 2-\mathrm{DMa}$ & -0.36483157 & 0.035183311 \\
\hline ENSMUSG00000091890 & A830073021Rik & -0.36175489 & 0.011968231 \\
\hline ENSMUSG00000083327 & Vcp-rs & -0.361193389 & 0.008325311 \\
\hline ENSMUSG00000020911 & Krt19 & -0.360532345 & 0.025384835 \\
\hline ENSMUSG00000043639 & Rbm20 & -0.358514829 & 0.004938199 \\
\hline ENSMUSG00000028447 & Dctn3 & -0.358475068 & 3.54E-05 \\
\hline ENSMUSG00000036073 & Galt & -0.358319934 & 0.000709789 \\
\hline ENSMUSG00000078727 & Gm2542 & -0.35560714 & 0.023357824 \\
\hline ENSMUSG00000039457 & Ppl & -0.347810887 & 0.005002175 \\
\hline ENSMUSG00000036114 & 2810432D09Rik & -0.346478055 & $2.11 \mathrm{E}-05$ \\
\hline ENSMUSG00000086180 & Rmst & -0.342215289 & 0.012717115 \\
\hline ENSMUSG00000078746 & Gm13298 & -0.336072322 & 0.031630909 \\
\hline ENSMUSG00000028393 & Alad & -0.335187017 & 0.002193137 \\
\hline ENSMUSG00000035451 & Foxa1 & -0.334889071 & 0.013207033 \\
\hline ENSMUSG00000040536 & Necab1 & -0.327347544 & 0.031856674 \\
\hline ENSMUSG00000086796 & 4932702P03Rik & -0.321666574 & 0.014966049 \\
\hline ENSMUSG00000051397 & Tacstd2 & -0.319453391 & 0.029637862 \\
\hline ENSMUSG00000026107 & Obfc2a & -0.31300041 & 0.024603458 \\
\hline ENSMUSG00000073889 & Il11ra1 & -0.307002006 & 0.001566128 \\
\hline ENSMUSG00000042448 & Hoxd1 & -0.306611254 & 0.04328941 \\
\hline ENSMUSG00000045591 & Olig3 & -0.305054297 & 0.023278691 \\
\hline ENSMUSG00000037369 & Kdm6a & -0.304827298 & 0.011985765 \\
\hline ENSMUSG00000084941 & Gm11944 & -0.304758751 & 0.047579588 \\
\hline
\end{tabular}




\begin{tabular}{llll} 
ENSMUSG00000062785 & Kcnc3 & -0.302012229 & 0.022765758 \\
ENSMUSG00000019845 & Tube1 & -0.301628925 & 0.008287381 \\
ENSMUSG00000022790 & lgsf11 & -0.300830029 & 0.030328379 \\
ENSMUSG00000042564 & 4933432 B09Rik & -0.300756901 & 0.048734933 \\
ENSMUSG00000042985 & Upk3b & -0.298811587 & 0.036736019 \\
ENSMUSG00000047965 & Rpl9-ps7 & -0.295016302 & 0.009639905 \\
ENSMUSG00000070282 & 3000002C10Rik & -0.291815924 & 0.020409602 \\
ENSMUSG00000019913 & Sim1 & -0.287834164 & 0.011229479 \\
ENSMUSG00000041930 & BC057022 & -0.284038412 & 0.011750179 \\
ENSMUSG00000086370 & B230206F22Rik & -0.28135244 & 0.036810276 \\
ENSMUSG00000092315 & Gm20440 & -0.276850894 & 0.022301176 \\
ENSMUSG00000046341 & Gm11223 & -0.274585873 & 0.022273274 \\
ENSMUSG00000000690 & Hoxb6 & -0.26718011 & 0.009502245 \\
ENSMUSG00000038253 & Hoxa5 & -0.265256538 & 0.000531584 \\
ENSMUSG00000085133 & B930095G15Rik & -0.263275388 & 0.045735525 \\
\hline
\end{tabular}

